CRYSTALLOGRAPHIC COMMUNICATIONS

ISSN 2056-9890

Received 7 February 2018

Accepted 26 February 2018

Edited by J. Ellena, Universidade de Sâo Paulo, Brazil

Keywords: crystal structure; cholesterol; gemini; analogue; hydrogen bonding.

CCDC reference: 1825767

Supporting information: this article has supporting information at journals.iucr.org/e

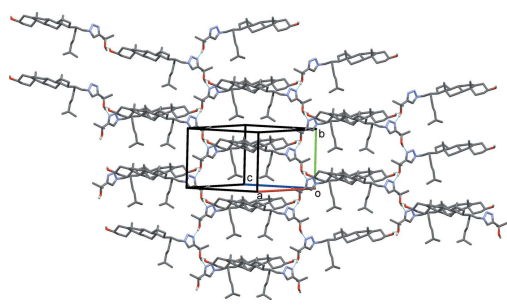

OPEN Ә ACCESS

\section{Crystal structure of (20S)-21-[4-(2-hydroxypropan- 2-yl)-1H-1,2,3-triazol-4-yl]-20-(4-methylpentyl\}-5- pregnen-3 $\beta$-ol with an unknown solvate}

\author{
Hugo Santalla $^{\mathrm{a} *}$ and Saray Argibay ${ }^{\mathrm{b}}$
}

a Departamento de Química Orgánica, Instituto de Investigación Sanitaria Galicia Sur, Facultade de Química, Universidade de Vigo, E-36310, Vigo, Spain, and ${ }^{\mathbf{b}}$ Departamento de Química Inorgánica, Instituto de Investigación Sanitaria Galicia Sur, Facultade de Química, Universidade de Vigo, E-36310, Vigo, Spain. *Correspondence e-mail: hsantalla@uvigo.es

In the title cholesterol analogue, [systematic name: $(3 S, 8 S, 9 S, 10 R, 13 S, 14 S, 17 R)$ 17-\{(S)-1-[4-(2-hydroxypropan-2-yl)-1H-1,2,3-triazol-1-yl]-6-methylheptan-2yl\}-10,13-dimethyl-2,3,4,7,8,9,10,11,12,13,14,15,16,17-tetradecahydro- $1 H$-cyclopenta[a]phenanthren-3-ol] $\mathrm{C}_{32} \mathrm{H}_{53} \mathrm{~N}_{3} \mathrm{O}_{2}$, a new chain, including an intermediate triazole and a tertiary hydroxyl group in the terminal position, has been added at position 20 inducing a change in its stereochemistry. In the crystal, molecules are linked by $\mathrm{O}-\mathrm{H} \cdots \mathrm{O}$ and $\mathrm{O}-\mathrm{H} \cdots \mathrm{N}$ hydrogen bonds, forming layers lying parallel to $(\overline{2} 01)$ and enclosing $R_{4}^{4}(36)$ ring motifs. The isopropyl group is disordered about two positions with a refined occupancy ratio of 0.763 (5):0.237 (5). A region of disordered electron density was corrected for using the SQUEEZE routine in PLATON (Spek (2015). Acta Cryst. C71, 9-18). The given chemical formula and other crystal data do not take into account the unknown solvent molecule(s).

\section{Chemical context}

The nuclear receptors (NRs) are a large family of ligandregulated transcriptional factors and include the receptors for steroid hormones, thyroid hormones, lipophilic vitamins and cholesterol metabolites (Mangelsdorf \& Evans, 1995; Burris et al., 2013). Approximately half of NRs are classified as orphan NRs because they do not have well-characterized ligands (Hummasti \& Tontonoz, 2008). Orphan NRs are an active area of research partly due to their potential for clinical agent development for various diseases (Mohan \& Heyman, 2003). Recent studies have demonstrated that retinoic acid receptorrelated orphan receptors (RORs) have been implicated in several physiological and pathological processes.

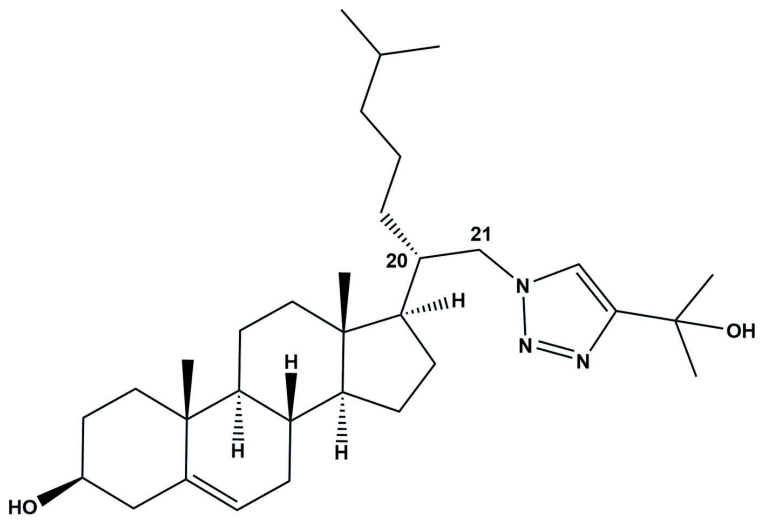




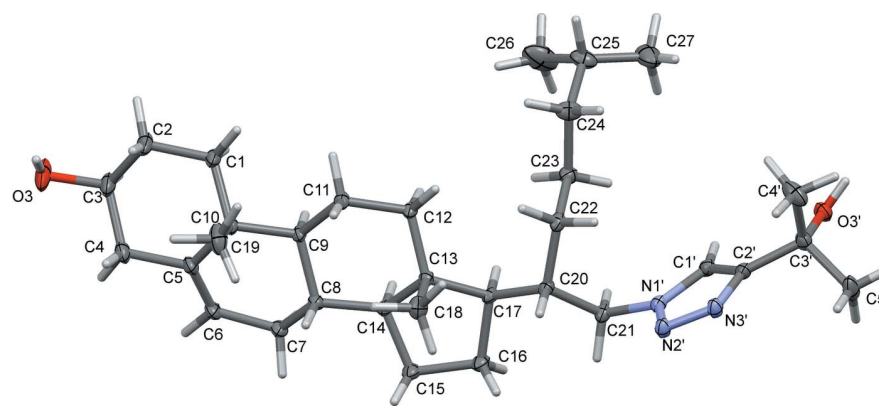

Figure 1

The molecular structure of compound $\mathbf{2}$, with the atom labelling. Displacement ellipsoids are drawn at the $30 \%$ probability level. In this and other figures the minor disorder component atoms $(\mathrm{C} 24 B-\mathrm{C} 27 B)$ of the aliphatic chain at $\mathrm{C} 20$ have been omitted for clarity.

Using the methodology developed in our research group for the synthesis of gemini-type vitamin D analogues (Fall et al., 2011; Pazos et al., 2016; Santalla et al., 2017) (modified with a double side chain), we can access new cholesterol analogues that can be of great interest in interactions with RORs. In this study, we present the structure of a new analogue of cholesterol (2), with eight stereocentres and a double side chain based on the aliphatic chain of cholesterol on the one hand and on the incorporation of a triazole ring on the other, since many azasteroids have proven to be biologically active. For example, some of them act as $5 \alpha$-reductase inhibitors, antifungal agents and $\gamma$-aminobutyric acid (GABA) receptor modulators (Tian et al., 1995; Burbiel \& Bracher, 2003; Covey et al., 2000).

\section{Structural commentary}

In the title cholesterol gemini-type analogue $\mathbf{2}$, illustrated in Fig. 1, the four aliphatic rings are structurally identical to those in the cholesterol hormone, $i$-cholesteryl methyl ether (Bernal et al., 1940; Wang et al., 2014). In the title compound, atom C20 has a different stereochemistry than in the cholesterol molecule, as a result of stereospecific reactions of the synthetic pathway. Furthermore, a new chain, including an intermediate triazole and a tertiary hydroxyl group in the terminal position, has been added at atom C21. Although some steroid analogues with a triazole ring have been synthesized (Seck et al., 2015), there are no references to any crystallographic analyses of gemini cholesterols with a triazole group at position C21 (Cambridge Structural Database, version 5.39, last update February 2018; Groom et al., 2016). The terminal OH group
Table 1

Hydrogen-bond geometry $\left(\AA{ }^{\circ}\right)$.

\begin{tabular}{lllll}
\hline$D-\mathrm{H} \cdots A$ & $D-\mathrm{H}$ & $\mathrm{H} \cdots A$ & $D \cdots A$ & $D-\mathrm{H} \cdots A$ \\
\hline $\mathrm{O}^{2}-\mathrm{H} \cdots \mathrm{O}^{\prime i}$ & 0.84 & 2.00 & $2.811(3)$ & 162 \\
$\mathrm{O}^{\prime}-\mathrm{H}^{\prime} \cdots \mathrm{N}^{\prime}{ }^{\text {ii }}$ & 0.84 & 1.97 & $2.810(2)$ & 175 \\
\hline
\end{tabular}

Symmetry codes: (i) $x+\frac{1}{2}, y+\frac{1}{2}, z+1$; (ii) $-x-\frac{1}{2}, y-\frac{1}{2},-z$.

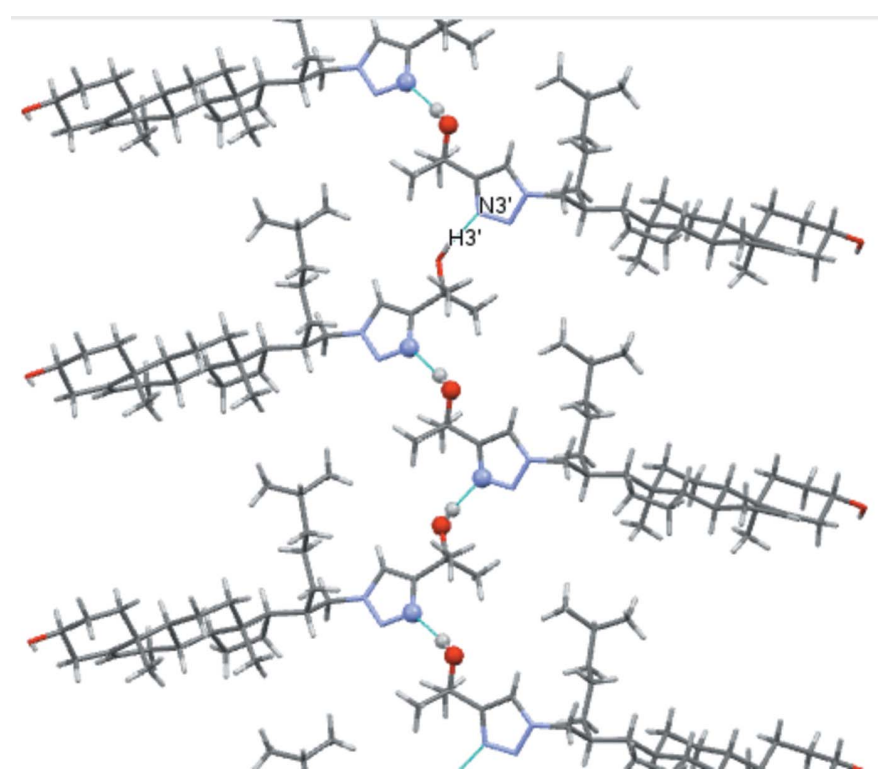

Figure 3

A view of the $\mathrm{O}-\mathrm{H} \cdots \mathrm{N}$ hydrogen bonded $C(5)$ chain propagating along the [010] direction (blue dashed lines; see Table 1).

$\left(\mathrm{C}^{\prime} / \mathrm{C}^{\prime} / \mathrm{O}^{\prime}\right)$ is inclined to the triazole ring $\left(\mathrm{N}^{\prime}-\mathrm{N}^{\prime} / \mathrm{Cl}^{\prime} / \mathrm{C2}^{\prime}\right)$ mean plane by $7.2(2)^{\circ}$.

\section{Supramolecular features}

The molecular association in the title compound $\mathbf{2}$, is based on hydrogen bonding involving the hydroxyl and triazole groups (Table 1). These intermolecular links are present in the form of two chains. The first, a $C(18)$ chain (Fig. 2), is formed by the $\mathrm{O} 3-\mathrm{H} 3 \cdots \mathrm{O}^{\prime \mathrm{i}}$ hydrogen bond with $\mathrm{O} 3-\mathrm{H} 3$ acting as the donor and atom $\mathrm{O}^{\prime}$ acting as the acceptor. The second is a $C(5)$ chain, in which the triazole group participates, and is formed by hydrogen bond $\mathrm{O}^{\prime}-\mathrm{H}^{\prime} \ldots \mathrm{N} 3^{\text {'ii }}$ (Fig. 3); the alcohol group $\mathrm{O}^{\prime}-\mathrm{H}^{\prime}$ acts as the donor towards the acceptor atom $\mathrm{N}^{\prime}$. The combination of these interactions

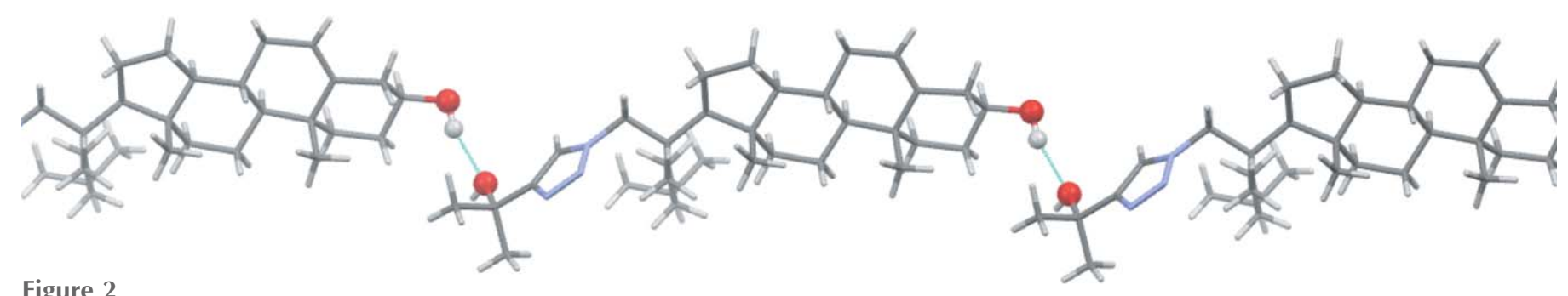

Figure 2

A view of the $\mathrm{O}-\mathrm{H} \cdots \mathrm{O}$ hydrogen bonded $C(18)$ chain propagating along the [102] direction (blue dashed lines; see Table 1). 


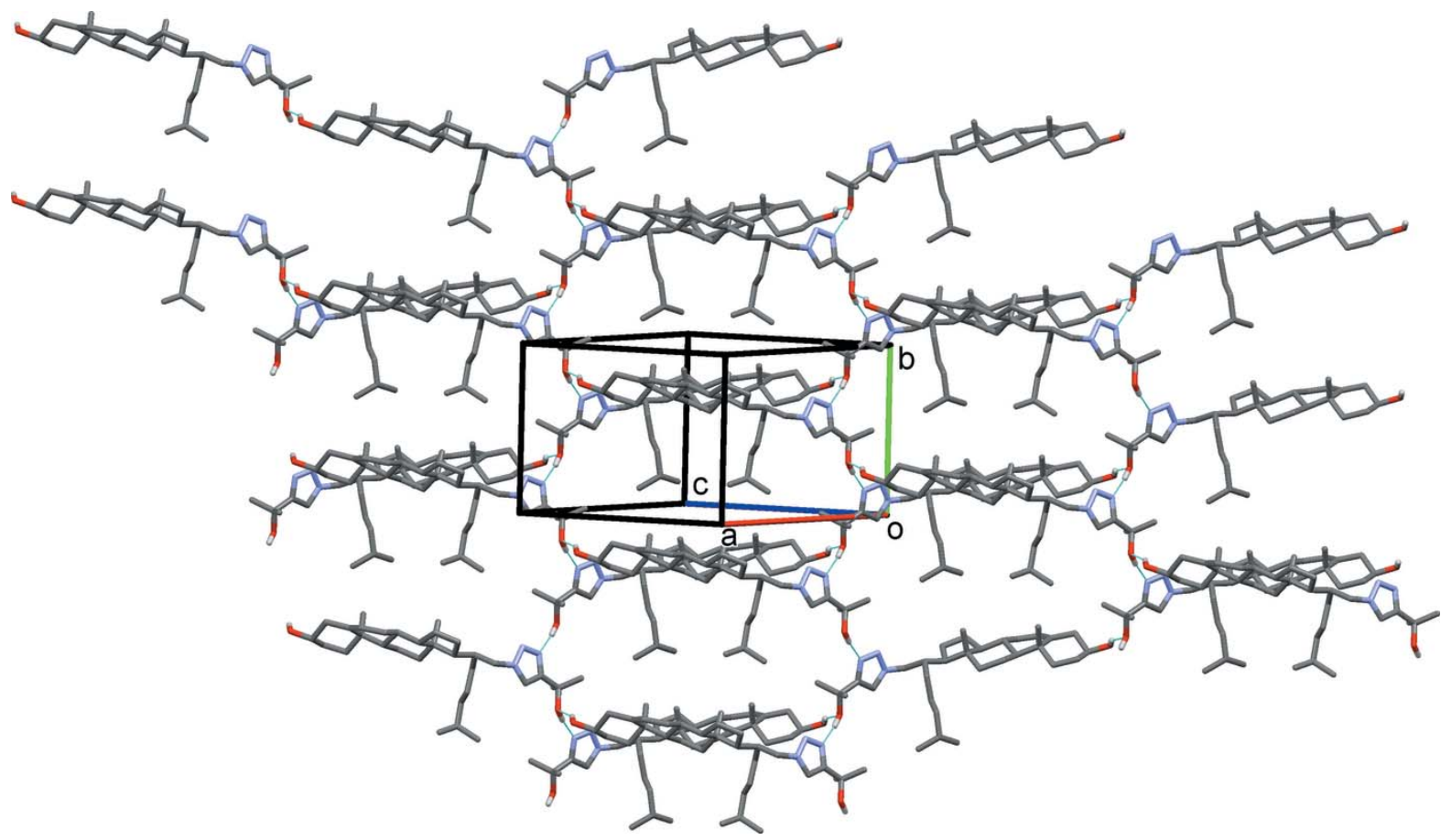

Figure 4

A view approximately normal to the (201) plane of the crystal packing of compound 2 . Hydrogen bonds (see Table 1 ) are shown as dashed lines, and only $\mathrm{H}$ atoms $\mathrm{H} 3$ and $\mathrm{H}^{\prime}$ have been included.

results in the formation of layers lying parallel to the $(\overline{2} 01)$ plane, as shown in Fig. 4, and encloses $R_{4}^{4}(36)$ ring motifs, details of which are illustrated in Fig. 5.

\section{Synthesis and crystallization}

Compound 2: details of the synthesis are illustrated in Fig. 6. To a solution of triazole 1 (12 mg, $0.022 \mathrm{mmol}$;) in ${ }^{t} \mathrm{BuOH}$ $(2 \mathrm{ml})$ and water $(1 \mathrm{ml})$ was added $p$-TsOH $(5 \mathrm{mg})$ and the mixture was heated to $353 \mathrm{~K}$ for $3 \mathrm{~h}$. The reaction mixture was diluted with water and then extracted with $\mathrm{CH}_{2} \mathrm{Cl}_{2}(3 \times 5 \mathrm{ml})$. The combined organic layers were dried with $\mathrm{Na}_{2} \mathrm{SO}_{4}$, filtered, and concentrated. The residue was purified by flash column chromatography (50\% EtOAc/hexane) to afford the title diol (11 $\mathrm{mg}, 99 \%)$. Compound 2 was recrystallized as colourless prisms by slow evaporation of a solvent mixture of dichloro- methane/diethyl ether (1:1) at room temperature [yield 99\%; m.p. $778 \mathrm{~K} ; R_{\mathrm{f}}: 0.10$ (30\% EtOAc/hexane)].

Spectroscopic data for 2: MS-ESI $[\mathrm{m} / \mathrm{z}(\%)]: 534.40(10)$ $\left[M^{+}+\mathrm{Na}\right], 512.42(100)\left[M^{+}+\mathrm{H}\right], 494.41(31)\left[M^{+}-\mathrm{OMe}\right] .{ }^{1} \mathrm{H}$ NMR $\left(\mathrm{CDCl}_{3}, \delta\right): 7.36\left(1 \mathrm{H}, s, \mathrm{H}-1^{\prime}\right), 5.35(1 \mathrm{H}, s, \mathrm{H}-6), 4.32$ $(1 \mathrm{H}, m, \mathrm{H}-21), 4.23(1 \mathrm{H}, m, \mathrm{H}-21), 3.52(1 \mathrm{H}, m, \mathrm{H}-3), 2.26(3 \mathrm{H}$, $m), 1.94(5 \mathrm{H}, m), 1.83(5 \mathrm{H}, m), 1.48(7 \mathrm{H}, m), 1.27(4 \mathrm{H}, m), 1.23$ $\left(6 \mathrm{H}, d, J=9.2 \mathrm{~Hz}, \mathrm{CH}_{3}-4^{\prime} / 5^{\prime}\right), 1.06(3 \mathrm{H}, m), 1.00\left(3 \mathrm{H}, s, \mathrm{CH}_{3}-\right.$ 18), $0.84\left(6 \mathrm{H}, d, J=6.6 \mathrm{~Hz}, \mathrm{CH}_{3}-26 / 27\right), 0.73\left(6 \mathrm{H}, s, \mathrm{CH}_{3}-19\right)$ ppm. ${ }^{13} \mathrm{C}$ NMR $\left(\mathrm{CDCl}_{3}, \delta\right): 140.74(\mathrm{C}-5), 128.78\left(\mathrm{C}-2^{\prime}\right), 121.51$ (CH-6), $112.41\left(\mathrm{C}-1^{\prime}\right), 77.20\left(\mathrm{C}-3^{\prime}\right), 71.73(\mathrm{CH}-3), 56.38(\mathrm{CH}-$ 14), $52.30\left(\mathrm{CH}_{2}-21\right), 50.25(\mathrm{CH}), 49.99(\mathrm{CH}), 42.73(\mathrm{C}-13)$, $42.23\left(\mathrm{CH}_{2}\right), 41.66(\mathrm{CH}), 39.20\left(\mathrm{CH}_{2}\right), 39.16\left(\mathrm{CH}_{2}\right), 37.23$ $\left(\mathrm{CH}_{2}\right), 36.48(\mathrm{C}-10), 31.93(\mathrm{CH}), 31.80\left(\mathrm{CH}_{2}\right), 31.61\left(\mathrm{CH}_{2}\right)$, $30.50\left(\mathrm{CH}_{3}-4^{\prime} / 5^{\prime}\right), 30.47\left(\mathrm{CH}_{3}-4^{\prime} / 5^{\prime}\right), 29.30\left(\mathrm{CH}_{2}\right), 27.85\left(\mathrm{CH}_{2}\right)$, $27.82(\mathrm{CH}), 24.26\left(\mathrm{CH}_{2}\right), 22.69\left(\mathrm{CH}_{3}-26 / 27\right), 22.52\left(\mathrm{CH}_{3}-26 /\right.$

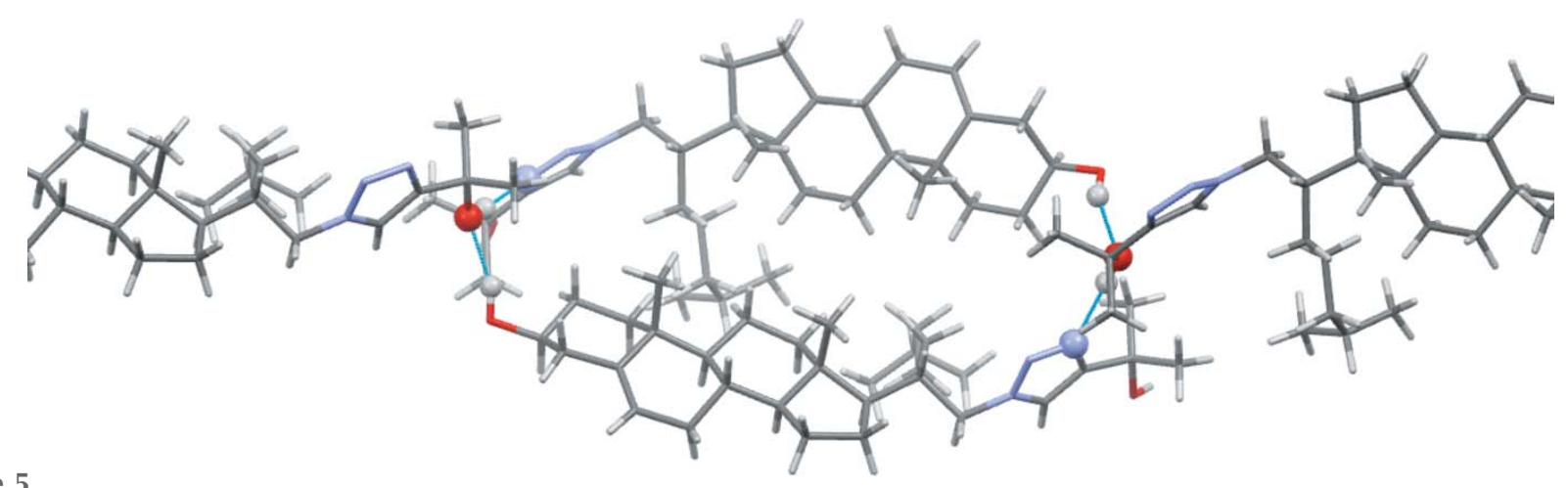

Figure 5

A partial view of the crystal packing of compound 2, showing details of the $\mathrm{O}-\mathrm{H} \cdots \mathrm{O}$ and $\mathrm{O}-\mathrm{H} \cdots \mathrm{N}$ hydrogen bonds forming an $R_{4}^{4}(36)$ ring motif (blue dashed lines; see Table 1). 

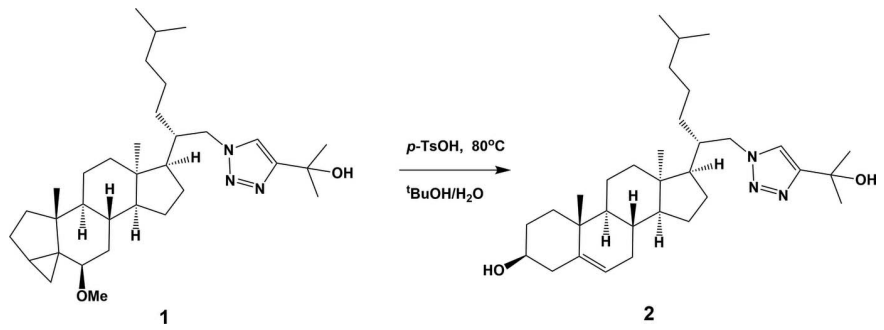

Figure 6

The synthesis of the title compound 2 .

27), $22.38\left(\mathrm{CH}_{2}\right), 21.07\left(\mathrm{CH}_{2}\right), 19.37\left(\mathrm{CH}_{3}-18\right), 12.08\left(\mathrm{CH}_{3}-19\right)$ p.p.m.

\section{Refinement}

Crystal data, data collection and structure refinement details are summarized in Table 2. The $\mathrm{O}-\mathrm{H}$ and $\mathrm{C}$-bound hydrogen atoms were positioned geometrically $(\mathrm{O}-\mathrm{H}=0.84 \AA, \mathrm{C}-\mathrm{H}=$ 0.95-1.00 ̊) and refined using a riding model with $U_{\text {iso }}(\mathrm{H})=$ $1.5 U_{\text {eq }}\left(\mathrm{O}\right.$-hydroxyl, C-methyl) and $1.2 U_{\text {eq }}(\mathrm{C})$ for other $\mathrm{H}$ atoms. The isopropyl group is disordered about two positions with a refined occupancy ratio of 0.763 (5):0.237 (5) for atoms C24-C27/C24B-C27B.

A region of disordered electron density was corrected for using the SQUEEZE routine in PLATON (Spek, 2015): volume ca $269 \AA^{3}$ for 96 electrons count per unit cell. There is possibly one molecule of diethyl ether per molecule of the title compound 2. The formula mass and unit-cell characteristics were not taken into account during refinement.

\section{Acknowledgements}

The work of the MS and X-ray divisions of the research support service of the University of Vigo (CACTI) is gratefully acknowledged.

\section{References}

Bernal, J. D., Crowfoot, D. \& Fankuchen, I. (1940). Philos. Trans. Roy. Soc. A: Math. Phys. Engineering Sci. 239, 135-182.

Bruker (2016). APEX3, SAINT and SADABS. Bruker AXS Inc., Madison, Wisconsin, USA.

Burbiel, J. \& Bracher, F. (2003). Steroids, 68, 587-594.

Burris, T. P., Solt, L. A., Wang, Y., Crumbley, C., Banerjee, S., Griffett, K., Lundasen, T., Hughes, T. \& Kojetin, D. J. (2013). Pharmacol. Rev. 65, 710-778.

Covey, D. F., Han, M., Kumar, A. S., de la Cruz, M. A. M., Meadows, E. S., Hu, Y., Tonnies, A., Nathan, D., Coleman, M., Benz, A., Evers, A. S., Zorumski, C. F. \& Mennerick, S. (2000). J. Med. Chem. 43, 3201-3204.

Dolomanov, O. V., Bourhis, L. J., Gildea, R. J., Howard, J. A. K. \& Puschmann, H. (2009). J. Appl. Cryst. 42, 339-341.

Fall, Y., Gómez, G., Pérez, M., Gándara, Z., Pérez, X., Pazos, G. \& Kurz, G. (2011). PCT Int. Appl. WO2011121152A120111006.

Groom, C. R., Bruno, I. J., Lightfoot, M. P. \& Ward, S. C. (2016). Acta Cryst. B72, 171-179.
Table 2

Experimental details.

Crystal data

Chemical formula

$M_{\mathrm{r}}$

Crystal system, space group

Temperature (K)

$a, b, c(\AA)$

$\beta\left({ }^{\circ}\right)$

$V\left(\AA^{3}\right)$

$Z$

Radiation type

$\mu\left(\mathrm{mm}^{-1}\right)$

Crystal size (mm)

$\mathrm{C}_{32} \mathrm{H}_{53} \mathrm{~N}_{3} \mathrm{O}$

511.77

Monoclinic, $C 2$

100

20.1130 (15), 10.3898 (7),

15.5934 (12)

$97.452(2)$

3231.0 (4)

4

Mo $K \alpha$

0.07

$0.35 \times 0.30 \times 0.24$

Data collection

Diffractometer

Absorption correction

$T_{\min }, T_{\max }$

No. of measured, independent and observed $[I>2 \sigma(I)]$ reflections

$R_{\text {int }}$
$(\sin \theta / \lambda)_{\max }\left(\AA^{-1}\right)$

Bruker D8 Venture Photon 100 CMOS

Multi-scan (SADABS; Bruker, 2016)

$0.688,0.746$

$85013,8040,7629$

0.029

0.670

Refinement

$R\left[F^{2}>2 \sigma\left(F^{2}\right)\right], w R\left(F^{2}\right), S$

No. of reflections

No. of parameters

No. of restraints

$\mathrm{H}$-atom treatment

$\Delta \rho_{\max }, \Delta \rho_{\min }\left(\mathrm{e} \AA^{-3}\right)$

Absolute structure

$0.044,0.122,1.02$

8040

363

5

$\mathrm{H}$-atom parameters constrained $0.46,-0.31$

Flack $x$ determined using 3430 quotients $\left[\left(I^{+}\right)-\left(I^{-}\right)\right] /\left[\left(I^{+}\right)+\left(I^{-}\right)\right]$ (Parsons et al., 2013)

Absolute structure parameter $0.1(3)$

Computer programs: $A P E X 3$ and SAINT (Bruker, 2016), SHELXT2014/5 (Sheldrick, 2015a), OLEX2 (Dolomanov et al., 2009), Mercury (Macrae et al., 2008), SHELXL2016/6 (Sheldrick, 2015b), PLATON (Spek, 2009) and publCIF (Westrip, 2010).

Hummasti, S. \& Tontonoz, P. (2008). Mol. Endocrinol. 22, 1743-1753. Macrae, C. F., Bruno, I. J., Chisholm, J. A., Edgington, P. R., McCabe, P., Pidcock, E., Rodriguez-Monge, L., Taylor, R., van de Streek, J. \& Wood, P. A. (2008). J. Appl. Cryst. 41, 466-470.

Mangelsdorf, D. J. \& Evans, R. M. (1995). Cell, 83, 841-850.

Mohan, R. \& Heyman, R. A. (2003). Curr. Top. Med. Chem. 3, $1637-$ 1647.

Parsons, S., Flack, H. D. \& Wagner, T. (2013). Acta Cryst. B69, 249259.

Pazos, G., Pérez, M., Gándara, Z., Gómez, G. \& Fall, Y. (2016). RSC Adv. 6, 61073-61076.

Santalla, H., Martínez, A., Garrido, F., Gómez, G. \& Fall, Y. (2017). Org. Chem. Front. 4, 1999-2001.

Seck, I., Fall, A., Lago, C., Sène, M., Gaye, M., Seck, M., Gómez, G. \& Fall, Y. (2015). Synthesis, 47, 2826-2830.

Sheldrick, G. M. (2015a). Acta Cryst. A71, 3-8.

Sheldrick, G. M. (2015b). Acta Cryst. C71, 3-8.

Spek, A. L. (2009). Acta Cryst. D65, 148-155.

Spek, A. L. (2015). Acta Cryst. C71, 9-18.

Tian, G., Mook, R., Moss, M. L. \& Frye, S. V. (1995). Biochemistry, 34, 13453-13459.

Wang, J.-R., Zhou, C., Yu, X. \& Mei, X. (2014). Chem. Commun. 50, 855-858.

Westrip, S. P. (2010). J. Appl. Cryst. 43, 920-925. 


\section{supporting information}

Acta Cryst. (2018). E74, 465-468 [https://doi.org/10.1107/S2056989018003286]

Crystal structure of (20S)-21-[4-(2-hydroxypropan-2-yl)-1H-1,2,3-triazol-4yl]-20-(4-methylpentyl)-5-pregnen-3 $\beta$-ol with an unknown solvate

Hugo Santalla and Saray Argibay

Computing details

Data collection: APEX3 (Bruker, 2016); cell refinement: SAINT (Bruker, 2016); data reduction: SAINT (Bruker, 2016); program(s) used to solve structure: SHELXT2014/5 (Sheldrick, 2015a); program(s) used to refine structure:

SHELXL2016/6 (Sheldrick, 2015b); molecular graphics: OLEX2 (Dolomanov et al., 2009) and Mercury (Macrae et al., 2008); software used to prepare material for publication: SHELXL2016/6 (Sheldrick, 2015b), PLATON (Spek, 2009) and publCIF (Westrip, 2010).

(20S)-21-[4-(2-Hydroxypropan-2-yl)-1H-1,2,3-triazol-4-yl]-20-(4-methylpentyl)-5-pregnen-3 $\beta$-ol

Crystal data

$\mathrm{C}_{32} \mathrm{H}_{53} \mathrm{~N}_{3} \mathrm{O}$

$M_{r}=511.77$

Monoclinic, $C 2$

$a=20.1130(15) \AA$

$b=10.3898$ (7) $\AA$

$c=15.5934(12) \AA$

$\beta=97.452(2)^{\circ}$

$V=3231.0(4) \AA^{3}$

$Z=4$

Data collection

Bruker D8 Venture Photon 100 CMOS diffractometer

$\varphi$ and $\omega$ scans

Absorption correction: multi-scan

(SADABS; Bruker, 2016)

$T_{\min }=0.688, T_{\max }=0.746$

85013 measured reflections

\section{Refinement}

Refinement on $F^{2}$

Least-squares matrix: full

$R\left[F^{2}>2 \sigma\left(F^{2}\right)\right]=0.044$

$w R\left(F^{2}\right)=0.122$

$S=1.02$

8040 reflections

363 parameters

5 restraints

Primary atom site location: structure-invariant direct methods
$F(000)=1128$

$D_{\mathrm{x}}=1.052 \mathrm{Mg} \mathrm{m}^{-3}$

Mo $K \alpha$ radiation, $\lambda=0.71073 \AA$

Cell parameters from 9096 reflections

$\theta=2.5-28.4^{\circ}$

$\mu=0.07 \mathrm{~mm}^{-1}$

$T=100 \mathrm{~K}$

Prism, colourless

$0.35 \times 0.30 \times 0.24 \mathrm{~mm}$

8040 independent reflections

7629 reflections with $I>2 \sigma(I)$

$R_{\text {int }}=0.029$

$\theta_{\text {max }}=28.4^{\circ}, \theta_{\text {min }}=2.5^{\circ}$

$h=-26 \rightarrow 26$

$k=-13 \rightarrow 13$

$l=-20 \rightarrow 20$

Secondary atom site location: difference Fourier map

Hydrogen site location: mixed

$\mathrm{H}$-atom parameters constrained

$w=1 /\left[\sigma^{2}\left(F_{\mathrm{o}}^{2}\right)+(0.0729 P)^{2}+1.6246 P\right]$

where $P=\left(F_{\mathrm{o}}{ }^{2}+2 F_{\mathrm{c}}{ }^{2}\right) / 3$

$(\Delta / \sigma)_{\max }=0.008$

$\Delta \rho_{\max }=0.46 \mathrm{e} \AA^{-3}$

$\Delta \rho_{\min }=-0.31$ e $\AA^{-3}$ 
Absolute structure: Flack $x$ determined using 3430 quotients $\left[\left(I^{+}\right)-\left(I^{-}\right)\right] /\left[\left(I^{+}\right)+\left(I^{-}\right)\right]$(Parsons et al., 2013)

Absolute structure parameter: $0.1(3)$

\section{Special details}

Geometry. All esds (except the esd in the dihedral angle between two 1.s. planes) are estimated using the full covariance matrix. The cell esds are taken into account individually in the estimation of esds in distances, angles and torsion angles; correlations between esds in cell parameters are only used when they are defined by crystal symmetry. An approximate (isotropic) treatment of cell esds is used for estimating esds involving l.s. planes.

Fractional atomic coordinates and isotropic or equivalent isotropic displacement parameters $\left(\AA^{2}\right)$

\begin{tabular}{|c|c|c|c|c|c|}
\hline & $x$ & $y$ & $z$ & $U_{\text {iso }} * / U_{\text {eq }}$ & Occ. $(<1)$ \\
\hline $\mathrm{O} 3$ & $0.35759(11)$ & $0.2665(4)$ & $0.82807(13)$ & $0.0775(9)$ & \\
\hline H3 & 0.329808 & 0.292008 & 0.860194 & $0.116^{*}$ & \\
\hline O3' & $-0.21535(8)$ & $-0.17225(15)$ & $-0.03536(11)$ & $0.0324(3)$ & \\
\hline $\mathrm{H} 3^{\prime}$ & -0.246827 & -0.224255 & -0.031655 & $0.049^{*}$ & \\
\hline $\mathrm{N} 1^{\prime}$ & $-0.07599(8)$ & $0.09363(17)$ & $0.07066(10)$ & $0.0246(3)$ & \\
\hline $\mathrm{N} 2^{\prime}$ & $-0.11583(9)$ & $0.19700(17)$ & $0.06509(12)$ & $0.0296(4)$ & \\
\hline N3' & $-0.17644(9)$ & $0.15741(18)$ & $0.03258(12)$ & $0.0291(4)$ & \\
\hline $\mathrm{C} 1$ & $0.21530(12)$ & $0.1479(3)$ & $0.66464(13)$ & $0.0399(5)$ & \\
\hline H1A & 0.168814 & 0.121675 & 0.670664 & $0.048^{*}$ & \\
\hline H1B & 0.238521 & 0.072198 & 0.643980 & $0.048^{*}$ & \\
\hline $\mathrm{C} 1^{\prime}$ & $-0.11028(11)$ & $-0.0135(2)$ & $0.04215(12)$ & $0.0257(4)$ & \\
\hline $\mathrm{H} 1^{\prime}$ & -0.093554 & -0.098775 & 0.039622 & $0.031^{*}$ & \\
\hline $\mathrm{C} 2$ & $0.25074(14)$ & $0.1869(4)$ & $0.75364(15)$ & $0.0538(8)$ & \\
\hline $\mathrm{H} 2 \mathrm{~A}$ & 0.226878 & 0.260248 & 0.776283 & $0.065^{*}$ & \\
\hline $\mathrm{H} 2 \mathrm{~B}$ & 0.250049 & 0.114006 & 0.794424 & $0.065^{*}$ & \\
\hline $\mathrm{C} 2^{\prime}$ & $-0.17475(10)$ & $0.02869(19)$ & $0.01766(12)$ & $0.0254(4)$ & \\
\hline $\mathrm{C} 3$ & $0.32250(13)$ & $0.2246(3)$ & $0.74675(15)$ & $0.0472(7)$ & \\
\hline $\mathrm{H} 3 \mathrm{~A}$ & 0.346192 & 0.147179 & 0.727638 & $0.057^{*}$ & \\
\hline $\mathrm{C} 3^{\prime}$ & $-0.23710(11)$ & $-0.0443(2)$ & $-0.01857(14)$ & $0.0305(4)$ & \\
\hline $\mathrm{C} 4$ & $0.32511(12)$ & $0.3296(3)$ & $0.67895(15)$ & $0.0388(5)$ & \\
\hline $\mathrm{H} 4 \mathrm{~A}$ & 0.307741 & 0.410699 & 0.700939 & $0.047^{*}$ & \\
\hline H4B & 0.372452 & 0.344304 & 0.670450 & $0.047^{*}$ & \\
\hline $\mathrm{C} 4^{\prime}$ & $-0.28702(14)$ & -0.0460 & $0.0473(2)$ & $0.0491(7)$ & \\
\hline $\mathrm{H} 4^{\prime} \mathrm{A}$ & -0.328751 & -0.087103 & 0.021575 & $0.074 *$ & \\
\hline $\mathrm{H} 4^{\prime} \mathrm{B}$ & -0.296399 & 0.042503 & 0.063909 & $0.074 *$ & \\
\hline $\mathrm{H} 4^{\prime} \mathrm{C}$ & -0.267970 & -0.094362 & 0.098575 & $0.074^{*}$ & \\
\hline $\mathrm{C} 5$ & $0.28505(10)$ & $0.2978(2)$ & $0.59230(13)$ & $0.0290(4)$ & \\
\hline $\mathrm{C} 5^{\prime}$ & $-0.26936(15)$ & $0.0171(3)$ & $-0.1032(2)$ & $0.0484(7)$ & \\
\hline H5'A & -0.236719 & 0.019264 & -0.144712 & $0.073^{*}$ & \\
\hline $\mathrm{H} 5^{\prime} \mathrm{B}$ & -0.283572 & 0.104994 & -0.091894 & $0.073^{*}$ & \\
\hline $\mathrm{H} 5^{\prime} \mathrm{C}$ & -0.308430 & -0.033800 & -0.127195 & $0.073^{*}$ & \\
\hline C6 & $0.31207(10)$ & $0.3053(2)$ & $0.51844(14)$ & $0.0313(4)$ & \\
\hline H6 & 0.358126 & 0.327892 & 0.522258 & $0.038^{*}$ & \\
\hline $\mathrm{C} 7$ & $0.27513(10)$ & $0.2807(2)$ & $0.43003(13)$ & $0.0300(4)$ & \\
\hline $\mathrm{H} 7 \mathrm{~A}$ & 0.289590 & 0.196862 & 0.408602 & $0.036^{*}$ & \\
\hline
\end{tabular}




\begin{tabular}{|c|c|c|c|c|c|}
\hline $\mathrm{H} 7 \mathrm{~B}$ & 0.286977 & 0.348379 & 0.389965 & $0.036^{*}$ & \\
\hline $\mathrm{C} 8$ & $0.19900(9)$ & $0.27924(19)$ & $0.42977(12)$ & $0.0231(4)$ & \\
\hline $\mathrm{H} 8$ & 0.182769 & 0.369334 & 0.435870 & $0.028^{*}$ & \\
\hline C9 & $0.18246(9)$ & $0.1983(2)$ & $0.50722(11)$ & $0.0223(3)$ & \\
\hline H9 & 0.204785 & 0.113112 & 0.502406 & $0.027^{*}$ & \\
\hline $\mathrm{C} 10$ & $0.21297(10)$ & $0.2561(2)$ & $0.59538(13)$ & $0.0275(4)$ & \\
\hline C11 & $0.10698(10)$ & $0.1703(2)$ & $0.50292(12)$ & $0.0293(4)$ & \\
\hline H11A & 0.100051 & 0.107618 & 0.548748 & $0.035^{*}$ & \\
\hline H11B & 0.083522 & 0.250810 & 0.514914 & $0.035^{*}$ & \\
\hline $\mathrm{C} 12$ & $0.07549(9)$ & $0.1166(2)$ & $0.41518(12)$ & $0.0257(4)$ & \\
\hline $\mathrm{H} 12 \mathrm{~A}$ & 0.026664 & 0.105256 & 0.415649 & $0.031 *$ & \\
\hline H12B & 0.095091 & 0.031154 & 0.405776 & $0.031^{*}$ & \\
\hline $\mathrm{C} 13$ & $0.08749(9)$ & $0.20702(19)$ & $0.34066(11)$ & $0.0222(3)$ & \\
\hline C14 & $0.16431(9)$ & $0.2218(2)$ & $0.34556(11)$ & $0.0237(4)$ & \\
\hline H14 & 0.182393 & 0.132477 & 0.342725 & $0.028 *$ & \\
\hline $\mathrm{C} 15$ & $0.17510(11)$ & $0.2859(2)$ & $0.26021(13)$ & $0.0320(4)$ & \\
\hline H15A & 0.219280 & 0.262394 & 0.243283 & $0.038^{*}$ & \\
\hline H15B & 0.172312 & 0.380801 & 0.264597 & $0.038^{*}$ & \\
\hline $\mathrm{C} 16$ & $0.11729(10)$ & $0.2325(2)$ & $0.19454(13)$ & $0.0300(4)$ & \\
\hline H16A & 0.135361 & 0.177939 & 0.150946 & $0.036^{*}$ & \\
\hline H16B & 0.091499 & 0.304031 & 0.164280 & $0.036^{*}$ & \\
\hline $\mathrm{C} 17$ & $0.07153(8)$ & $0.1516(2)$ & $0.24737(11)$ & $0.0212(3)$ & \\
\hline H17 & 0.088297 & 0.060868 & 0.248789 & $0.025^{*}$ & \\
\hline $\mathrm{C} 18$ & $0.05172(11)$ & $0.3366(2)$ & $0.34758(15)$ & $0.0333(5)$ & \\
\hline H18A & 0.056778 & 0.389264 & 0.296676 & $0.050 *$ & \\
\hline H18B & 0.071597 & 0.381738 & 0.399894 & $0.050 *$ & \\
\hline $\mathrm{H} 18 \mathrm{C}$ & 0.003982 & 0.321512 & 0.350577 & $0.050 *$ & \\
\hline $\mathrm{C} 19$ & $0.17159(14)$ & $0.3708(3)$ & $0.62128(19)$ & $0.0494(7)$ & \\
\hline H19A & 0.164581 & 0.432269 & 0.573271 & $0.074 *$ & \\
\hline H19B & 0.195719 & 0.413239 & 0.672214 & $0.074 *$ & \\
\hline H19C & 0.128087 & 0.340122 & 0.634875 & $0.074 *$ & \\
\hline $\mathrm{C} 20$ & $-0.00253(9)$ & $0.1494(2)$ & $0.20600(11)$ & $0.0231(3)$ & \\
\hline $\mathrm{H} 20$ & -0.019403 & 0.239995 & 0.205211 & $0.028^{*}$ & \\
\hline $\mathrm{C} 21$ & $-0.00603(9)$ & $0.1048(2)$ & $0.11160(12)$ & $0.0265(4)$ & \\
\hline $\mathrm{H} 21 \mathrm{~A}$ & 0.018056 & 0.167241 & 0.078850 & $0.032 *$ & \\
\hline $\mathrm{H} 21 \mathrm{~B}$ & 0.016486 & 0.020320 & 0.109684 & $0.032 *$ & \\
\hline $\mathrm{C} 22$ & $-0.04890(9)$ & $0.0678(2)$ & $0.25508(12)$ & $0.0255(4)$ & \\
\hline $\mathrm{H} 22 \mathrm{~A}$ & -0.048827 & 0.105022 & 0.313526 & $0.031 *$ & \\
\hline $\mathrm{H} 22 \mathrm{~B}$ & -0.095151 & 0.074933 & 0.224772 & $0.031 *$ & \\
\hline $\mathrm{C} 23$ & $-0.03115(10)$ & $-0.0746(2)$ & $0.26492(14)$ & $0.0304(4)$ & \\
\hline $\mathrm{H} 23 \mathrm{~A}$ & 0.016646 & -0.084771 & 0.288671 & $0.036^{*}$ & \\
\hline H23B & -0.038774 & -0.118011 & 0.207982 & $0.036^{*}$ & \\
\hline $\mathrm{C} 24$ & $-0.0767(2)$ & $-0.1347(4)$ & $0.3276(2)$ & $0.0389(7)$ & $0.763(5)$ \\
\hline $\mathrm{H} 24 \mathrm{~A}$ & -0.066712 & -0.092988 & 0.384918 & $0.047^{*}$ & $0.763(5)$ \\
\hline H24B & -0.124162 & -0.116555 & 0.305481 & $0.047^{*}$ & $0.763(5)$ \\
\hline $\mathrm{C} 25$ & $-0.06732(19)$ & $-0.2815(4)$ & 0.3385 & $0.0428(8)$ & $0.763(5)$ \\
\hline $\mathrm{H} 25$ & -0.093048 & -0.306183 & 0.386759 & $0.051^{*}$ & $0.763(5)$ \\
\hline $\mathrm{C} 26$ & $0.0043(3)$ & $-0.3176(4)$ & $0.3687(5)$ & $0.0768(17)$ & $0.763(5)$ \\
\hline
\end{tabular}




$\begin{array}{llllll}\text { H26A } & 0.023690 & -0.255904 & 0.412492 & 0.115^{*} & 0.763(5) \\ \text { H26B } & 0.030030 & -0.316282 & 0.319486 & 0.115^{*} & 0.763(5) \\ \text { H26C } & 0.005819 & -0.404206 & 0.393806 & 0.115^{*} & 0.763(5) \\ \text { C27 } & -0.0969(3) & -0.3579(8) & 0.2627(4) & 0.0667(14) & 0.763(5) \\ \text { H27A } & -0.069611 & -0.347475 & 0.215460 & 0.100^{*} & 0.763(5) \\ \text { H27B } & -0.142641 & -0.327817 & 0.243705 & 0.100^{*} & 0.763(5) \\ \text { H27C } & -0.098069 & -0.449028 & 0.278740 & 0.100^{*} & 0.763(5) \\ \text { C24B } & -0.0510(7) & -0.1677(11) & 0.3327(8) & 0.0389(7) & 0.237(5) \\ \text { H24B } & -0.028169 & -0.138464 & 0.389453 & 0.047^{*} & 0.237(5) \\ \text { H24A } & -0.099715 & -0.156564 & 0.334308 & 0.047^{*} & 0.237(5) \\ \text { C25B } & -0.0384(7) & -0.3100(11) & 0.3271(8) & 0.0428(8) & 0.237(5) \\ \text { H25 } & 0.008894 & -0.317481 & 0.314094 & 0.051^{*} & 0.237(5) \\ \text { C26B } & -0.0377(6) & -0.3632(17) & 0.4122(10) & 0.073(5) & 0.237(5) \\ \text { H26A } & -0.079316 & -0.340174 & 0.434835 & 0.109^{*} & 0.237(5) \\ \text { H26B } & 0.000683 & -0.328578 & 0.450387 & 0.109^{*} & 0.237(5) \\ \text { H26C } & -0.033923 & -0.457077 & 0.409337 & 0.109^{*} & 0.237(5) \\ \text { C27B } & -0.0815(13) & -0.359(3) & 0.2463(14) & 0.0667(14) & 0.237(5) \\ \text { H27A } & -0.098369 & -0.285928 & 0.210137 & 0.100^{*} & 0.237(5) \\ \text { H27B } & -0.119378 & -0.408162 & 0.262993 & 0.100^{*} & 0.237(5) \\ \text { H27C } & -0.054504 & -0.414775 & 0.213602 & 0.100^{*} & 0.237(5)\end{array}$

Atomic displacement parameters $\left(\AA^{2}\right)$

\begin{tabular}{lllllll}
\hline & $U^{11}$ & $U^{22}$ & $U^{33}$ & $U^{12}$ & $U^{13}$ & $U^{23}$ \\
\hline $\mathrm{O} 3^{3}$ & $0.0457(11)$ & $0.155(3)$ & $0.0296(9)$ & $-0.0415(15)$ & $-0.0044(8)$ & $-0.0143(13)$ \\
$\mathrm{O}^{\prime}$ & $0.0347(8)$ & $0.0252(7)$ & $0.0361(8)$ & $-0.0052(6)$ & $0.0007(6)$ & $-0.0033(6)$ \\
$\mathrm{N}^{\prime}$ & $0.0255(8)$ & $0.0276(8)$ & $0.0197(7)$ & $0.0004(7)$ & $-0.0010(6)$ & $0.0009(6)$ \\
$\mathrm{N}^{\prime}$ & $0.0305(9)$ & $0.0242(8)$ & $0.0308(8)$ & $0.0007(7)$ & $-0.0081(7)$ & $0.0003(7)$ \\
$\mathrm{N} 3^{\prime}$ & $0.0286(8)$ & $0.0239(8)$ & $0.0315(8)$ & $0.0002(7)$ & $-0.0087(7)$ & $0.0007(7)$ \\
$\mathrm{C} 1$ & $0.0368(11)$ & $0.0615(15)$ & $0.0200(9)$ & $-0.0227(11)$ & $-0.0010(8)$ & $0.0035(10)$ \\
C1' & $0.0320(10)$ & $0.0240(9)$ & $0.0206(8)$ & $0.0007(7)$ & $0.0009(7)$ & $-0.0010(7)$ \\
C2 & $0.0421(13)$ & $0.097(2)$ & $0.0215(10)$ & $-0.0329(14)$ & $-0.0009(9)$ & $0.0020(12)$ \\
C2' & $0.0296(10)$ & $0.0249(9)$ & $0.0205(8)$ & $-0.0011(7)$ & $-0.0017(7)$ & $0.0010(7)$ \\
C3 & $0.0368(12)$ & $0.079(2)$ & $0.0243(10)$ & $-0.0214(13)$ & $-0.0031(9)$ & $-0.0042(11)$ \\
C3' & $0.0305(10)$ & $0.0242(10)$ & $0.0351(10)$ & $-0.0036(8)$ & $-0.0025(8)$ & $-0.0032(8)$ \\
C4 & $0.0298(10)$ & $0.0524(14)$ & $0.0333(11)$ & $-0.0169(10)$ & $0.0006(8)$ & $-0.0112(10)$ \\
C4' & $0.0425(14)$ & $0.0390(13)$ & $0.0687(18)$ & $-0.0098(11)$ & $0.0181(13)$ & $-0.0177(13)$ \\
C5 & $0.0233(9)$ & $0.0337(11)$ & $0.0293(9)$ & $-0.0103(8)$ & $0.0010(7)$ & $-0.0031(8)$ \\
C5 & $0.0464(14)$ & $0.0374(13)$ & $0.0533(15)$ & $-0.0052(10)$ & $-0.0250(12)$ & $0.0007(11)$ \\
C6 & $0.0219(9)$ & $0.0403(12)$ & $0.0314(10)$ & $-0.0115(8)$ & $0.0020(7)$ & $-0.0001(8)$ \\
C7 & $0.0220(9)$ & $0.0415(11)$ & $0.0267(9)$ & $-0.0094(8)$ & $0.0042(7)$ & $0.0023(8)$ \\
C8 & $0.0203(8)$ & $0.0254(9)$ & $0.0237(8)$ & $-0.0046(7)$ & $0.0032(6)$ & $0.0016(7)$ \\
C9 & $0.0196(8)$ & $0.0296(9)$ & $0.0179(7)$ & $-0.0062(7)$ & $0.0032(6)$ & $-0.0027(7)$ \\
C10 & $0.0235(9)$ & $0.0367(11)$ & $0.0223(8)$ & $-0.0075(8)$ & $0.0032(7)$ & $-0.0060(8)$ \\
C11 & $0.0211(8)$ & $0.0483(13)$ & $0.0190(8)$ & $-0.0097(8)$ & $0.0048(6)$ & $-0.0028(8)$ \\
C12 & $0.0198(8)$ & $0.0396(11)$ & $0.0178(8)$ & $-0.0082(7)$ & $0.0029(6)$ & $0.0010(7)$ \\
C13 & $0.0190(8)$ & $0.0292(9)$ & $0.0184(7)$ & $-0.0006(7)$ & $0.0026(6)$ & $0.0003(7)$ \\
C14 & $0.0195(8)$ & $0.0331(10)$ & $0.0190(8)$ & $-0.0041(7)$ & $0.0041(6)$ & $0.0017(7)$ \\
& & & & & &
\end{tabular}


supporting information

\begin{tabular}{lllllll} 
& & & & \\
C15 & $0.0278(9)$ & $0.0457(12)$ & $0.0229(9)$ & $-0.0083(9)$ & $0.0040(7)$ & $0.0066(8)$ \\
C16 & $0.0257(9)$ & $0.0430(12)$ & $0.0215(8)$ & $-0.0027(8)$ & $0.0042(7)$ & $0.0063(8)$ \\
C17 & $0.0181(8)$ & $0.0287(9)$ & $0.0171(7)$ & $0.0013(7)$ & $0.0032(6)$ & $0.0018(7)$ \\
C18 & $0.0301(10)$ & $0.0339(11)$ & $0.0349(10)$ & $0.0067(9)$ & $0.0007(8)$ & $-0.0065(9)$ \\
C19 & $0.0378(13)$ & $0.0621(17)$ & $0.0473(14)$ & $0.0038(12)$ & $0.0023(11)$ & $-0.0304(13)$ \\
C20 & $0.0196(8)$ & $0.0314(9)$ & $0.0179(7)$ & $0.0026(7)$ & $0.0008(6)$ & $0.0004(7)$ \\
C21 & $0.0218(8)$ & $0.0377(10)$ & $0.0196(8)$ & $-0.0001(8)$ & $0.0015(6)$ & $-0.0010(8)$ \\
C22 & $0.0169(8)$ & $0.0391(11)$ & $0.0205(8)$ & $0.0018(7)$ & $0.0026(6)$ & $-0.0010(7)$ \\
C23 & $0.0230(9)$ & $0.0369(11)$ & $0.0312(10)$ & $-0.0016(8)$ & $0.0031(7)$ & $0.0063(8)$ \\
C24 & $0.037(2)$ & $0.0354(18)$ & $0.0487(16)$ & $0.0075(13)$ & $0.0199(15)$ & $0.0068(13)$ \\
C25 & $0.0342(19)$ & $0.0376(17)$ & $0.0599(19)$ & $-0.0024(14)$ & $0.0192(15)$ & $0.0051(14)$ \\
C26 & $0.057(3)$ & $0.043(2)$ & $0.129(5)$ & $0.0094(19)$ & $0.006(3)$ & $0.025(3)$ \\
C27 & $0.080(4)$ & $0.072(2)$ & $0.053(3)$ & $-0.037(3)$ & $0.029(2)$ & $-0.010(2)$ \\
C24B & $0.037(2)$ & $0.0354(18)$ & $0.0487(16)$ & $0.0075(13)$ & $0.0199(15)$ & $0.0068(13)$ \\
C25B & $0.0342(19)$ & $0.0376(17)$ & $0.0599(19)$ & $-0.0024(14)$ & $0.0192(15)$ & $0.0051(14)$ \\
C26B & $0.027(5)$ & $0.086(11)$ & $0.103(12)$ & $0.012(6)$ & $-0.003(6)$ & $-0.044(10)$ \\
C27B & $0.080(4)$ & $0.072(2)$ & $0.053(3)$ & $-0.037(3)$ & $0.029(2)$ & $-0.010(2)$ \\
& & & & & & \\
\hline
\end{tabular}

Geometric parameters $\left(\AA,{ }^{\circ}\right)$

\begin{tabular}{llll}
\hline $\mathrm{O} 3-\mathrm{C} 3$ & $1.437(3)$ & $\mathrm{C} 13-\mathrm{C} 17$ & $1.559(2)$ \\
$\mathrm{O} 3-\mathrm{H} 3$ & 0.8400 & $\mathrm{C} 14-\mathrm{C} 15$ & $1.529(3)$ \\
$\mathrm{O} 3^{\prime}-\mathrm{C} 3^{\prime}$ & $1.434(3)$ & $\mathrm{C} 14-\mathrm{H} 14$ & 1.0000 \\
$\mathrm{O} 3^{\prime}-\mathrm{H} 3^{\prime}$ & 0.8400 & $\mathrm{C} 15-\mathrm{C} 16$ & $1.549(3)$ \\
$\mathrm{N} 1^{\prime}-\mathrm{N} 2^{\prime}$ & $1.336(2)$ & $\mathrm{C} 15-\mathrm{H} 15 \mathrm{~A}$ & 0.9900 \\
$\mathrm{~N} 1^{\prime}-\mathrm{C} 1^{\prime}$ & $1.354(3)$ & $\mathrm{C} 15-\mathrm{H} 15 \mathrm{~B}$ & 0.9900 \\
$\mathrm{~N} 1^{\prime}-\mathrm{C} 21$ & $1.472(2)$ & $\mathrm{C} 16-\mathrm{C} 17$ & $1.559(3)$ \\
$\mathrm{N} 2^{\prime}-\mathrm{N} 3^{\prime}$ & $1.324(2)$ & $\mathrm{C} 16-\mathrm{H} 16 \mathrm{~A}$ & 0.9900 \\
$\mathrm{~N} 3^{\prime}-\mathrm{C} 2^{\prime}$ & $1.359(3)$ & $\mathrm{C} 16-\mathrm{H} 16 \mathrm{~B}$ & 0.9900 \\
$\mathrm{C} 1-\mathrm{C} 2$ & $1.530(3)$ & $\mathrm{C} 17-\mathrm{C} 20$ & $1.545(2)$ \\
$\mathrm{C} 1-\mathrm{C} 10$ & $1.555(3)$ & $\mathrm{C} 17-\mathrm{H} 17$ & 1.0000 \\
$\mathrm{C} 1-\mathrm{H} 1 \mathrm{~A}$ & 0.9900 & $\mathrm{C} 18-\mathrm{H} 18 \mathrm{~A}$ & 0.9800 \\
$\mathrm{C} 1-\mathrm{H} 1 \mathrm{~B}$ & 0.9900 & $\mathrm{C} 18-\mathrm{H} 18 \mathrm{~B}$ & 0.9800 \\
$\mathrm{C} 1^{\prime}-\mathrm{C} 2^{\prime}$ & $1.375(3)$ & $\mathrm{C} 18-\mathrm{H} 18 \mathrm{C}$ & 0.9800 \\
$\mathrm{C} 1^{\prime}-\mathrm{H} 1^{\prime}$ & 0.9500 & $\mathrm{C} 19-\mathrm{H} 19 \mathrm{~A}$ & 0.9800 \\
$\mathrm{C} 2-\mathrm{C} 3$ & $1.513(3)$ & $\mathrm{C} 19-\mathrm{H} 19 \mathrm{~B}$ & 0.9800 \\
$\mathrm{C} 2-\mathrm{H} 2 \mathrm{~A}$ & 0.9900 & $\mathrm{C} 19-\mathrm{H} 19 \mathrm{C}$ & 0.9800 \\
$\mathrm{C} 2-\mathrm{H} 2 \mathrm{~B}$ & 0.9900 & $\mathrm{C} 20-\mathrm{C} 21$ & $1.536(3)$ \\
$\mathrm{C} 2^{\prime}-\mathrm{C} 3^{\prime}$ & $1.511(3)$ & $\mathrm{C} 20-\mathrm{C} 22$ & $1.536(3)$ \\
$\mathrm{C} 3-\mathrm{C} 4$ & $1.525(4)$ & $\mathrm{C} 20-\mathrm{H} 20$ & 1.0000 \\
$\mathrm{C} 3-\mathrm{H} 3 \mathrm{~A}$ & 1.0000 & $\mathrm{C} 21-\mathrm{H} 21 \mathrm{~A}$ & 0.9900 \\
$\mathrm{C} 3^{\prime}-\mathrm{C} 4^{\prime}$ & $1.526(4)$ & $\mathrm{C} 21-\mathrm{H} 21 \mathrm{~B}$ & 0.9900 \\
$\mathrm{C} 3^{\prime}-\mathrm{C} 5^{\prime}$ & $1.532(3)$ & $\mathrm{C} 22-\mathrm{C} 23$ & $1.525(3)$ \\
$\mathrm{C} 4-\mathrm{C} 5$ & $1.517(3)$ & $\mathrm{C} 22-\mathrm{H} 22 \mathrm{~A}$ & 0.9900 \\
$\mathrm{C} 4-\mathrm{H} 4 \mathrm{~A}$ & 0.9900 & $\mathrm{C} 22-\mathrm{H} 22 \mathrm{~B}$ & 0.9900 \\
$\mathrm{C} 4-\mathrm{H} 4 \mathrm{~B}$ & 0.9900 & $\mathrm{C} 23-\mathrm{C} 24 \mathrm{~B}$ & $1.524(11)$ \\
$\mathrm{C} 4{ }^{\prime}-\mathrm{H} 4^{\prime} \mathrm{A}$ & 0.9800 & $\mathrm{C} 23-\mathrm{C} 24$ & 0.9901 \\
$\mathrm{C} 4{ }^{\prime}-\mathrm{H} 4{ }^{\prime} \mathrm{B}$ & 0.9800 & $\mathrm{C} 23-\mathrm{H} 23 \mathrm{~A}$ &
\end{tabular}




\begin{tabular}{|c|c|c|c|}
\hline $\mathrm{C} 4^{\prime}-\mathrm{H} 4^{\prime} \mathrm{C}$ & 0.9800 & $\mathrm{C} 23-\mathrm{H} 23 \mathrm{~B}$ & 0.9899 \\
\hline $\mathrm{C} 5-\mathrm{C} 6$ & $1.338(3)$ & $\mathrm{C} 24-\mathrm{C} 25$ & $1.544(5)$ \\
\hline $\mathrm{C} 5-\mathrm{C} 10$ & $1.520(3)$ & $\mathrm{C} 24-\mathrm{H} 24 \mathrm{~A}$ & 0.9900 \\
\hline $\mathrm{C} 5^{\prime}-\mathrm{H} 5^{\prime} \mathrm{A}$ & 0.9800 & $\mathrm{C} 24-\mathrm{H} 24 \mathrm{~B}$ & 0.9900 \\
\hline $\mathrm{C} 5^{\prime}-\mathrm{H} 5^{\prime} \mathrm{B}$ & 0.9800 & $\mathrm{C} 25-\mathrm{C} 27$ & $1.484(7)$ \\
\hline $\mathrm{C} 5^{\prime}-\mathrm{H} 5^{\prime} \mathrm{C}$ & 0.9800 & $\mathrm{C} 25-\mathrm{C} 26$ & $1.504(6)$ \\
\hline $\mathrm{C} 6-\mathrm{C} 7$ & $1.501(3)$ & $\mathrm{C} 25-\mathrm{H} 25$ & 1.0000 \\
\hline $\mathrm{C} 6-\mathrm{H} 6$ & 0.9500 & $\mathrm{C} 26-\mathrm{H} 26 \mathrm{~A}$ & 0.9800 \\
\hline $\mathrm{C} 7-\mathrm{C} 8$ & $1.531(3)$ & $\mathrm{C} 26-\mathrm{H} 26 \mathrm{~B}$ & 0.9800 \\
\hline C7-H7A & 0.9900 & $\mathrm{C} 26-\mathrm{H} 26 \mathrm{C}$ & 0.9800 \\
\hline C7-H7B & 0.9900 & $\mathrm{C} 27-\mathrm{H} 27 \mathrm{~A}$ & 0.9800 \\
\hline $\mathrm{C} 8-\mathrm{C} 14$ & $1.526(3)$ & $\mathrm{C} 27-\mathrm{H} 27 \mathrm{~B}$ & 0.9800 \\
\hline $\mathrm{C} 8-\mathrm{C} 9$ & $1.543(3)$ & $\mathrm{C} 27-\mathrm{H} 27 \mathrm{C}$ & 0.9800 \\
\hline $\mathrm{C} 8-\mathrm{H} 8$ & 1.0000 & $\mathrm{C} 24 \mathrm{~B}-\mathrm{C} 25 \mathrm{~B}$ & $1.504(15)$ \\
\hline $\mathrm{C} 9-\mathrm{C} 11$ & $1.539(3)$ & $\mathrm{C} 24 \mathrm{~B}-\mathrm{H} 24 \mathrm{~B}$ & 0.9900 \\
\hline $\mathrm{C} 9-\mathrm{C} 10$ & $1.552(2)$ & $\mathrm{C} 24 \mathrm{~B}-\mathrm{H} 24 \mathrm{~A}$ & 0.9900 \\
\hline C9-H9 & 1.0000 & $\mathrm{C} 25 \mathrm{~B}-\mathrm{C} 26 \mathrm{~B}$ & $1.436(19)$ \\
\hline $\mathrm{C} 10-\mathrm{C} 19$ & $1.538(3)$ & $\mathrm{C} 25 \mathrm{~B}-\mathrm{C} 27 \mathrm{~B}$ & $1.52(2)$ \\
\hline $\mathrm{C} 11-\mathrm{C} 12$ & $1.536(3)$ & $\mathrm{C} 25 \mathrm{~B}-\mathrm{H} 25$ & 1.0000 \\
\hline $\mathrm{C} 11-\mathrm{H} 11 \mathrm{~A}$ & 0.9900 & $\mathrm{C} 26 \mathrm{~B}-\mathrm{H} 26 \mathrm{~A}$ & 0.9800 \\
\hline $\mathrm{C} 11-\mathrm{H} 11 \mathrm{~B}$ & 0.9900 & $\mathrm{C} 26 \mathrm{~B}-\mathrm{H} 26 \mathrm{~B}$ & 0.9800 \\
\hline $\mathrm{C} 12-\mathrm{C} 13$ & $1.537(3)$ & $\mathrm{C} 26 \mathrm{~B}-\mathrm{H} 26 \mathrm{C}$ & 0.9800 \\
\hline $\mathrm{C} 12-\mathrm{H} 12 \mathrm{~A}$ & 0.9900 & $\mathrm{C} 27 \mathrm{~B}-\mathrm{H} 27 \mathrm{~A}$ & 0.9800 \\
\hline $\mathrm{C} 12-\mathrm{H} 12 \mathrm{~B}$ & 0.9900 & $\mathrm{C} 27 \mathrm{~B}-\mathrm{H} 27 \mathrm{~B}$ & 0.9800 \\
\hline $\mathrm{C} 13-\mathrm{C} 18$ & $1.537(3)$ & $\mathrm{C} 27 \mathrm{~B}-\mathrm{H} 27 \mathrm{C}$ & 0.9800 \\
\hline $\mathrm{C} 13-\mathrm{C} 14$ & $1.545(2)$ & & \\
\hline $\mathrm{C} 3-\mathrm{O} 3-\mathrm{H} 3$ & 109.5 & $\mathrm{C} 15-\mathrm{C} 14-\mathrm{H} 14$ & 105.8 \\
\hline $\mathrm{C} 3^{\prime}-\mathrm{O}^{\prime}-\mathrm{H} 3^{\prime}$ & 109.5 & $\mathrm{C} 13-\mathrm{C} 14-\mathrm{H} 14$ & 105.8 \\
\hline $\mathrm{N} 2^{\prime}-\mathrm{N} 1^{\prime}-\mathrm{C} 1^{\prime}$ & $111.34(16)$ & $\mathrm{C} 14-\mathrm{C} 15-\mathrm{C} 16$ & $103.90(16)$ \\
\hline $\mathrm{N} 2^{\prime}-\mathrm{N} 1^{\prime}-\mathrm{C} 21$ & $119.75(17)$ & $\mathrm{C} 14-\mathrm{C} 15-\mathrm{H} 15 \mathrm{~A}$ & 111.0 \\
\hline $\mathrm{C} 1^{\prime}-\mathrm{N} 1^{\prime}-\mathrm{C} 21$ & $128.66(18)$ & $\mathrm{C} 16-\mathrm{C} 15-\mathrm{H} 15 \mathrm{~A}$ & 111.0 \\
\hline $\mathrm{N} 3^{\prime}-\mathrm{N} 2^{\prime}-\mathrm{N} 1^{\prime}$ & $106.85(16)$ & $\mathrm{C} 14-\mathrm{C} 15-\mathrm{H} 15 \mathrm{~B}$ & 111.0 \\
\hline $\mathrm{N} 2^{\prime}-\mathrm{N} 3^{\prime}-\mathrm{C} 2^{\prime}$ & $109.22(17)$ & $\mathrm{C} 16-\mathrm{C} 15-\mathrm{H} 15 \mathrm{~B}$ & 111.0 \\
\hline $\mathrm{C} 2-\mathrm{C} 1-\mathrm{C} 10$ & $114.0(2)$ & $\mathrm{H} 15 \mathrm{~A}-\mathrm{C} 15-\mathrm{H} 15 \mathrm{~B}$ & 109.0 \\
\hline $\mathrm{C} 2-\mathrm{C} 1-\mathrm{H} 1 \mathrm{~A}$ & 108.7 & $\mathrm{C} 15-\mathrm{C} 16-\mathrm{C} 17$ & $106.74(15)$ \\
\hline $\mathrm{C} 10-\mathrm{C} 1-\mathrm{H} 1 \mathrm{~A}$ & 108.7 & $\mathrm{C} 15-\mathrm{C} 16-\mathrm{H} 16 \mathrm{~A}$ & 110.4 \\
\hline $\mathrm{C} 2-\mathrm{C} 1-\mathrm{H} 1 \mathrm{~B}$ & 108.7 & $\mathrm{C} 17-\mathrm{C} 16-\mathrm{H} 16 \mathrm{~A}$ & 110.4 \\
\hline $\mathrm{C} 10-\mathrm{C} 1-\mathrm{H} 1 \mathrm{~B}$ & 108.7 & $\mathrm{C} 15-\mathrm{C} 16-\mathrm{H} 16 \mathrm{~B}$ & 110.4 \\
\hline $\mathrm{H} 1 \mathrm{~A}-\mathrm{C} 1-\mathrm{H} 1 \mathrm{~B}$ & 107.6 & $\mathrm{C} 17-\mathrm{C} 16-\mathrm{H} 16 \mathrm{~B}$ & 110.4 \\
\hline $\mathrm{N} 1^{\prime}-\mathrm{C} 1^{\prime}-\mathrm{C} 2^{\prime}$ & $104.51(18)$ & $\mathrm{H} 16 \mathrm{~A}-\mathrm{C} 16-\mathrm{H} 16 \mathrm{~B}$ & 108.6 \\
\hline $\mathrm{N} 1^{\prime}-\mathrm{C} 1^{\prime}-\mathrm{H} 1^{\prime}$ & 127.7 & $\mathrm{C} 20-\mathrm{C} 17-\mathrm{C} 16$ & $112.98(15)$ \\
\hline $\mathrm{C} 2^{\prime}-\mathrm{C} 1^{\prime}-\mathrm{H} 1^{\prime}$ & 127.7 & $\mathrm{C} 20-\mathrm{C} 17-\mathrm{C} 13$ & $117.75(14)$ \\
\hline $\mathrm{C} 3-\mathrm{C} 2-\mathrm{C} 1$ & $109.9(2)$ & $\mathrm{C} 16-\mathrm{C} 17-\mathrm{C} 13$ & $103.26(15)$ \\
\hline $\mathrm{C} 3-\mathrm{C} 2-\mathrm{H} 2 \mathrm{~A}$ & 109.7 & $\mathrm{C} 20-\mathrm{C} 17-\mathrm{H} 17$ & 107.4 \\
\hline $\mathrm{C} 1-\mathrm{C} 2-\mathrm{H} 2 \mathrm{~A}$ & 109.7 & $\mathrm{C} 16-\mathrm{C} 17-\mathrm{H} 17$ & 107.4 \\
\hline $\mathrm{C} 3-\mathrm{C} 2-\mathrm{H} 2 \mathrm{~B}$ & 109.7 & $\mathrm{C} 13-\mathrm{C} 17-\mathrm{H} 17$ & 107.4 \\
\hline $\mathrm{C} 1-\mathrm{C} 2-\mathrm{H} 2 \mathrm{~B}$ & 109.7 & $\mathrm{C} 13-\mathrm{C} 18-\mathrm{H} 18 \mathrm{~A}$ & 109.5 \\
\hline
\end{tabular}




\begin{tabular}{|c|c|c|c|}
\hline $\mathrm{H} 2 \mathrm{~A}-\mathrm{C} 2-\mathrm{H} 2 \mathrm{~B}$ & 108.2 & $\mathrm{C} 13-\mathrm{C} 18-\mathrm{H} 18 \mathrm{~B}$ & 109.5 \\
\hline $\mathrm{N} 3^{\prime}-\mathrm{C} 2^{\prime}-\mathrm{C} 1^{\prime}$ & $108.07(18)$ & $\mathrm{H} 18 \mathrm{~A}-\mathrm{C} 18-\mathrm{H} 18 \mathrm{~B}$ & 109.5 \\
\hline $\mathrm{N} 3^{\prime}-\mathrm{C} 2^{\prime}-\mathrm{C} 3^{\prime}$ & $121.33(19)$ & $\mathrm{C} 13-\mathrm{C} 18-\mathrm{H} 18 \mathrm{C}$ & 109.5 \\
\hline $\mathrm{C} 1^{\prime}-\mathrm{C} 2^{\prime}-\mathrm{C} 3^{\prime}$ & $130.60(19)$ & $\mathrm{H} 18 \mathrm{~A}-\mathrm{C} 18-\mathrm{H} 18 \mathrm{C}$ & 109.5 \\
\hline $\mathrm{O} 3-\mathrm{C} 3-\mathrm{C} 2$ & $112.3(2)$ & $\mathrm{H} 18 \mathrm{~B}-\mathrm{C} 18-\mathrm{H} 18 \mathrm{C}$ & 109.5 \\
\hline $\mathrm{O} 3-\mathrm{C} 3-\mathrm{C} 4$ & $109.8(2)$ & $\mathrm{C} 10-\mathrm{C} 19-\mathrm{H} 19 \mathrm{~A}$ & 109.5 \\
\hline $\mathrm{C} 2-\mathrm{C} 3-\mathrm{C} 4$ & $110.7(2)$ & $\mathrm{C} 10-\mathrm{C} 19-\mathrm{H} 19 \mathrm{~B}$ & 109.5 \\
\hline $\mathrm{O} 3-\mathrm{C} 3-\mathrm{H} 3 \mathrm{~A}$ & 108.0 & $\mathrm{H} 19 \mathrm{~A}-\mathrm{C} 19-\mathrm{H} 19 \mathrm{~B}$ & 109.5 \\
\hline $\mathrm{C} 2-\mathrm{C} 3-\mathrm{H} 3 \mathrm{~A}$ & 108.0 & $\mathrm{C} 10-\mathrm{C} 19-\mathrm{H} 19 \mathrm{C}$ & 109.5 \\
\hline $\mathrm{C} 4-\mathrm{C} 3-\mathrm{H} 3 \mathrm{~A}$ & 108.0 & $\mathrm{H} 19 \mathrm{~A}-\mathrm{C} 19-\mathrm{H} 19 \mathrm{C}$ & 109.5 \\
\hline $\mathrm{O} 3^{\prime}-\mathrm{C} 3^{\prime}-\mathrm{C} 2^{\prime}$ & $105.98(17)$ & $\mathrm{H} 19 \mathrm{~B}-\mathrm{C} 19-\mathrm{H} 19 \mathrm{C}$ & 109.5 \\
\hline $\mathrm{O} 3^{\prime}-\mathrm{C} 3^{\prime}-\mathrm{C} 4^{\prime}$ & $110.79(19)$ & $\mathrm{C} 21-\mathrm{C} 20-\mathrm{C} 22$ & $110.83(17)$ \\
\hline $\mathrm{C} 2^{\prime}-\mathrm{C} 3^{\prime}-\mathrm{C} 4^{\prime}$ & $109.92(19)$ & $\mathrm{C} 21-\mathrm{C} 20-\mathrm{C} 17$ & 109.05 (14) \\
\hline $\mathrm{O} 3^{\prime}-\mathrm{C} 3^{\prime}-\mathrm{C} 5^{\prime}$ & $109.48(19)$ & $\mathrm{C} 22-\mathrm{C} 20-\mathrm{C} 17$ & $114.50(15)$ \\
\hline $\mathrm{C} 2^{\prime}-\mathrm{C} 3^{\prime}-\mathrm{C} 5^{\prime}$ & $110.59(19)$ & $\mathrm{C} 21-\mathrm{C} 20-\mathrm{H} 20$ & 107.4 \\
\hline $\mathrm{C} 4^{\prime}-\mathrm{C} 3^{\prime}-\mathrm{C} 5^{\prime}$ & $110.0(2)$ & $\mathrm{C} 22-\mathrm{C} 20-\mathrm{H} 20$ & 107.4 \\
\hline $\mathrm{C} 5-\mathrm{C} 4-\mathrm{C} 3$ & $113.54(19)$ & $\mathrm{C} 17-\mathrm{C} 20-\mathrm{H} 20$ & 107.4 \\
\hline $\mathrm{C} 5-\mathrm{C} 4-\mathrm{H} 4 \mathrm{~A}$ & 108.9 & $\mathrm{~N} 1{ }^{\prime}-\mathrm{C} 21-\mathrm{C} 20$ & $111.20(15)$ \\
\hline $\mathrm{C} 3-\mathrm{C} 4-\mathrm{H} 4 \mathrm{~A}$ & 108.9 & $\mathrm{~N} 1{ }^{\prime}-\mathrm{C} 21-\mathrm{H} 21 \mathrm{~A}$ & 109.4 \\
\hline $\mathrm{C} 5-\mathrm{C} 4-\mathrm{H} 4 \mathrm{~B}$ & 108.9 & $\mathrm{C} 20-\mathrm{C} 21-\mathrm{H} 21 \mathrm{~A}$ & 109.4 \\
\hline $\mathrm{C} 3-\mathrm{C} 4-\mathrm{H} 4 \mathrm{~B}$ & 108.9 & $\mathrm{~N} 1{ }^{\prime}-\mathrm{C} 21-\mathrm{H} 21 \mathrm{~B}$ & 109.4 \\
\hline $\mathrm{H} 4 \mathrm{~A}-\mathrm{C} 4-\mathrm{H} 4 \mathrm{~B}$ & 107.7 & $\mathrm{C} 20-\mathrm{C} 21-\mathrm{H} 21 \mathrm{~B}$ & 109.4 \\
\hline $\mathrm{C} 3^{\prime}-\mathrm{C} 4^{\prime}-\mathrm{H} 4^{\prime} \mathrm{A}$ & 109.5 & $\mathrm{H} 21 \mathrm{~A}-\mathrm{C} 21-\mathrm{H} 21 \mathrm{~B}$ & 108.0 \\
\hline $\mathrm{C} 3^{\prime}-\mathrm{C} 4^{\prime}-\mathrm{H} 4^{\prime} \mathrm{B}$ & 109.5 & $\mathrm{C} 23-\mathrm{C} 22-\mathrm{C} 20$ & $115.88(16)$ \\
\hline $\mathrm{H} 4^{\prime} \mathrm{A}-\mathrm{C} 4^{\prime}-\mathrm{H} 4^{\prime} \mathrm{B}$ & 109.5 & $\mathrm{C} 23-\mathrm{C} 22-\mathrm{H} 22 \mathrm{~A}$ & 108.3 \\
\hline $\mathrm{C} 3^{\prime}-\mathrm{C} 4^{\prime}-\mathrm{H} 4^{\prime} \mathrm{C}$ & 109.5 & $\mathrm{C} 20-\mathrm{C} 22-\mathrm{H} 22 \mathrm{~A}$ & 108.3 \\
\hline $\mathrm{H} 4^{\prime} \mathrm{A}-\mathrm{C} 4^{\prime}-\mathrm{H} 4^{\prime} \mathrm{C}$ & 109.5 & $\mathrm{C} 23-\mathrm{C} 22-\mathrm{H} 22 \mathrm{~B}$ & 108.3 \\
\hline $\mathrm{H} 4^{\prime} \mathrm{B}-\mathrm{C} 4^{\prime}-\mathrm{H} 4^{\prime} \mathrm{C}$ & 109.5 & $\mathrm{C} 20-\mathrm{C} 22-\mathrm{H} 22 \mathrm{~B}$ & 108.3 \\
\hline $\mathrm{C} 6-\mathrm{C} 5-\mathrm{C} 4$ & $121.72(18)$ & $\mathrm{H} 22 \mathrm{~A}-\mathrm{C} 22-\mathrm{H} 22 \mathrm{~B}$ & 107.4 \\
\hline $\mathrm{C} 6-\mathrm{C} 5-\mathrm{C} 10$ & $122.64(18)$ & $\mathrm{C} 24 \mathrm{~B}-\mathrm{C} 23-\mathrm{C} 22$ & $127.3(5)$ \\
\hline $\mathrm{C} 4-\mathrm{C} 5-\mathrm{C} 10$ & $115.64(18)$ & $\mathrm{C} 22-\mathrm{C} 23-\mathrm{C} 24$ & $107.7(2)$ \\
\hline $\mathrm{C} 3^{\prime}-\mathrm{C} 5^{\prime}-\mathrm{H} 5^{\prime} \mathrm{A}$ & 109.5 & $\mathrm{C} 24 \mathrm{~B}-\mathrm{C} 23-\mathrm{H} 23 \mathrm{~A}$ & 90.1 \\
\hline $\mathrm{C} 3^{\prime}-\mathrm{C} 5^{\prime}-\mathrm{H} 5^{\prime} \mathrm{B}$ & 109.5 & $\mathrm{C} 22-\mathrm{C} 23-\mathrm{H} 23 \mathrm{~A}$ & 110.2 \\
\hline $\mathrm{H} 5^{\prime} \mathrm{A}-\mathrm{C} 5^{\prime}-\mathrm{H} 5^{\prime} \mathrm{B}$ & 109.5 & $\mathrm{C} 24-\mathrm{C} 23-\mathrm{H} 23 \mathrm{~A}$ & 110.2 \\
\hline $\mathrm{C} 3^{\prime}-\mathrm{C} 5^{\prime}-\mathrm{H} 5^{\prime} \mathrm{C}$ & 109.5 & $\mathrm{C} 24 \mathrm{~B}-\mathrm{C} 23-\mathrm{H} 23 \mathrm{~B}$ & 108.0 \\
\hline $\mathrm{H} 5^{\prime} \mathrm{A}-\mathrm{C} 5^{\prime}-\mathrm{H} 5^{\prime} \mathrm{C}$ & 109.5 & $\mathrm{C} 22-\mathrm{C} 23-\mathrm{H} 23 \mathrm{~B}$ & 110.2 \\
\hline $\mathrm{H} 5^{\prime} \mathrm{B}-\mathrm{C} 5^{\prime}-\mathrm{H} 5^{\prime} \mathrm{C}$ & 109.5 & $\mathrm{C} 24-\mathrm{C} 23-\mathrm{H} 23 \mathrm{~B}$ & 110.2 \\
\hline $\mathrm{C} 5-\mathrm{C} 6-\mathrm{C} 7$ & $124.95(18)$ & $\mathrm{H} 23 \mathrm{~A}-\mathrm{C} 23-\mathrm{H} 23 \mathrm{~B}$ & 108.5 \\
\hline $\mathrm{C} 5-\mathrm{C} 6-\mathrm{H} 6$ & 117.5 & $\mathrm{C} 25-\mathrm{C} 24-\mathrm{C} 23$ & $113.1(3)$ \\
\hline $\mathrm{C} 7-\mathrm{C} 6-\mathrm{H} 6$ & 117.5 & $\mathrm{C} 25-\mathrm{C} 24-\mathrm{H} 24 \mathrm{~A}$ & 109.0 \\
\hline $\mathrm{C} 6-\mathrm{C} 7-\mathrm{C} 8$ & $112.33(17)$ & $\mathrm{C} 23-\mathrm{C} 24-\mathrm{H} 24 \mathrm{~A}$ & 109.0 \\
\hline $\mathrm{C} 6-\mathrm{C} 7-\mathrm{H} 7 \mathrm{~A}$ & 109.1 & $\mathrm{C} 25-\mathrm{C} 24-\mathrm{H} 24 \mathrm{~B}$ & 109.0 \\
\hline $\mathrm{C} 8-\mathrm{C} 7-\mathrm{H} 7 \mathrm{~A}$ & 109.1 & $\mathrm{C} 23-\mathrm{C} 24-\mathrm{H} 24 \mathrm{~B}$ & 109.0 \\
\hline $\mathrm{C} 6-\mathrm{C} 7-\mathrm{H} 7 \mathrm{~B}$ & 109.1 & $\mathrm{H} 24 \mathrm{~A}-\mathrm{C} 24-\mathrm{H} 24 \mathrm{~B}$ & 107.8 \\
\hline $\mathrm{C} 8-\mathrm{C} 7-\mathrm{H} 7 \mathrm{~B}$ & 109.1 & $\mathrm{C} 27-\mathrm{C} 25-\mathrm{C} 26$ & $112.6(5)$ \\
\hline $\mathrm{H} 7 \mathrm{~A}-\mathrm{C} 7-\mathrm{H} 7 \mathrm{~B}$ & 107.9 & $\mathrm{C} 27-\mathrm{C} 25-\mathrm{C} 24$ & $114.2(5)$ \\
\hline $\mathrm{C} 14-\mathrm{C} 8-\mathrm{C} 7$ & $110.59(16)$ & $\mathrm{C} 26-\mathrm{C} 25-\mathrm{C} 24$ & $112.3(3)$ \\
\hline $\mathrm{C} 14-\mathrm{C} 8-\mathrm{C} 9$ & $109.85(15)$ & $\mathrm{C} 27-\mathrm{C} 25-\mathrm{H} 25$ & 105.6 \\
\hline
\end{tabular}




\begin{tabular}{|c|c|c|c|}
\hline $\mathrm{C} 7-\mathrm{C} 8-\mathrm{C} 9$ & $108.69(16)$ & $\mathrm{C} 26-\mathrm{C} 25-\mathrm{H} 25$ & 105.6 \\
\hline $\mathrm{C} 14-\mathrm{C} 8-\mathrm{H} 8$ & 109.2 & $\mathrm{C} 24-\mathrm{C} 25-\mathrm{H} 25$ & 105.6 \\
\hline $\mathrm{C} 7-\mathrm{C} 8-\mathrm{H} 8$ & 109.2 & $\mathrm{C} 25-\mathrm{C} 26-\mathrm{H} 26 \mathrm{~A}$ & 109.5 \\
\hline $\mathrm{C} 9-\mathrm{C} 8-\mathrm{H} 8$ & 109.2 & $\mathrm{C} 25-\mathrm{C} 26-\mathrm{H} 26 \mathrm{~B}$ & 109.5 \\
\hline $\mathrm{C} 11-\mathrm{C} 9-\mathrm{C} 8$ & $112.38(15)$ & $\mathrm{H} 26 \mathrm{~A}-\mathrm{C} 26-\mathrm{H} 26 \mathrm{~B}$ & 109.5 \\
\hline $\mathrm{C} 11-\mathrm{C} 9-\mathrm{C} 10$ & $112.73(15)$ & $\mathrm{C} 25-\mathrm{C} 26-\mathrm{H} 26 \mathrm{C}$ & 109.5 \\
\hline $\mathrm{C} 8-\mathrm{C} 9-\mathrm{C} 10$ & $112.47(15)$ & $\mathrm{H} 26 \mathrm{~A}-\mathrm{C} 26-\mathrm{H} 26 \mathrm{C}$ & 109.5 \\
\hline $\mathrm{C} 11-\mathrm{C} 9-\mathrm{H} 9$ & 106.2 & $\mathrm{H} 26 \mathrm{~B}-\mathrm{C} 26-\mathrm{H} 26 \mathrm{C}$ & 109.5 \\
\hline $\mathrm{C} 8-\mathrm{C} 9-\mathrm{H} 9$ & 106.2 & $\mathrm{C} 25-\mathrm{C} 27-\mathrm{H} 27 \mathrm{~A}$ & 109.5 \\
\hline $\mathrm{C} 10-\mathrm{C} 9-\mathrm{H} 9$ & 106.2 & $\mathrm{C} 25-\mathrm{C} 27-\mathrm{H} 27 \mathrm{~B}$ & 109.5 \\
\hline $\mathrm{C} 5-\mathrm{C} 10-\mathrm{C} 19$ & $109.78(19)$ & $\mathrm{H} 27 \mathrm{~A}-\mathrm{C} 27-\mathrm{H} 27 \mathrm{~B}$ & 109.5 \\
\hline $\mathrm{C} 5-\mathrm{C} 10-\mathrm{C} 9$ & $110.59(15)$ & $\mathrm{C} 25-\mathrm{C} 27-\mathrm{H} 27 \mathrm{C}$ & 109.5 \\
\hline $\mathrm{C} 19-\mathrm{C} 10-\mathrm{C} 9$ & $111.55(18)$ & $\mathrm{H} 27 \mathrm{~A}-\mathrm{C} 27-\mathrm{H} 27 \mathrm{C}$ & 109.5 \\
\hline $\mathrm{C} 5-\mathrm{C} 10-\mathrm{C} 1$ & $106.57(17)$ & $\mathrm{H} 27 \mathrm{~B}-\mathrm{C} 27-\mathrm{H} 27 \mathrm{C}$ & 109.5 \\
\hline $\mathrm{C} 19-\mathrm{C} 10-\mathrm{C} 1$ & $110.3(2)$ & $\mathrm{C} 25 \mathrm{~B}-\mathrm{C} 24 \mathrm{~B}-\mathrm{C} 23$ & $121.4(9)$ \\
\hline $\mathrm{C} 9-\mathrm{C} 10-\mathrm{C} 1$ & $107.97(17)$ & $\mathrm{C} 25 \mathrm{~B}-\mathrm{C} 24 \mathrm{~B}-\mathrm{H} 24 \mathrm{~B}$ & 107.0 \\
\hline $\mathrm{C} 12-\mathrm{C} 11-\mathrm{C} 9$ & $113.46(15)$ & $\mathrm{C} 23-\mathrm{C} 24 \mathrm{~B}-\mathrm{H} 24 \mathrm{~B}$ & 107.0 \\
\hline $\mathrm{C} 12-\mathrm{C} 11-\mathrm{H} 11 \mathrm{~A}$ & 108.9 & $\mathrm{C} 25 \mathrm{~B}-\mathrm{C} 24 \mathrm{~B}-\mathrm{H} 24 \mathrm{~A}$ & 107.0 \\
\hline $\mathrm{C} 9-\mathrm{C} 11-\mathrm{H} 11 \mathrm{~A}$ & 108.9 & $\mathrm{C} 23-\mathrm{C} 24 \mathrm{~B}-\mathrm{H} 24 \mathrm{~A}$ & 107.0 \\
\hline $\mathrm{C} 12-\mathrm{C} 11-\mathrm{H} 11 \mathrm{~B}$ & 108.9 & $\mathrm{H} 24 \mathrm{~B}-\mathrm{C} 24 \mathrm{~B}-\mathrm{H} 24 \mathrm{~A}$ & 106.7 \\
\hline C9-C11-H11B & 108.9 & $\mathrm{C} 26 \mathrm{~B}-\mathrm{C} 25 \mathrm{~B}-\mathrm{C} 24 \mathrm{~B}$ & $107.8(12)$ \\
\hline $\mathrm{H} 11 \mathrm{~A}-\mathrm{C} 11-\mathrm{H} 11 \mathrm{~B}$ & 107.7 & $\mathrm{C} 26 \mathrm{~B}-\mathrm{C} 25 \mathrm{~B}-\mathrm{C} 27 \mathrm{~B}$ & $124.9(14)$ \\
\hline $\mathrm{C} 11-\mathrm{C} 12-\mathrm{C} 13$ & $111.36(16)$ & $\mathrm{C} 24 \mathrm{~B}-\mathrm{C} 25 \mathrm{~B}-\mathrm{C} 27 \mathrm{~B}$ & $107.2(17)$ \\
\hline $\mathrm{C} 11-\mathrm{C} 12-\mathrm{H} 12 \mathrm{~A}$ & 109.4 & $\mathrm{C} 26 \mathrm{~B}-\mathrm{C} 25 \mathrm{~B}-\mathrm{H} 25$ & 105.1 \\
\hline $\mathrm{C} 13-\mathrm{C} 12-\mathrm{H} 12 \mathrm{~A}$ & 109.4 & $\mathrm{C} 24 \mathrm{~B}-\mathrm{C} 25 \mathrm{~B}-\mathrm{H} 25$ & 105.1 \\
\hline $\mathrm{C} 11-\mathrm{C} 12-\mathrm{H} 12 \mathrm{~B}$ & 109.4 & $\mathrm{C} 27 \mathrm{~B}-\mathrm{C} 25 \mathrm{~B}-\mathrm{H} 25$ & 105.1 \\
\hline $\mathrm{C} 13-\mathrm{C} 12-\mathrm{H} 12 \mathrm{~B}$ & 109.4 & $\mathrm{C} 25 \mathrm{~B}-\mathrm{C} 26 \mathrm{~B}-\mathrm{H} 26 \mathrm{~A}$ & 109.5 \\
\hline $\mathrm{H} 12 \mathrm{~A}-\mathrm{C} 12-\mathrm{H} 12 \mathrm{~B}$ & 108.0 & $\mathrm{C} 25 \mathrm{~B}-\mathrm{C} 26 \mathrm{~B}-\mathrm{H} 26 \mathrm{~B}$ & 109.5 \\
\hline $\mathrm{C} 18-\mathrm{C} 13-\mathrm{C} 12$ & $111.19(16)$ & $\mathrm{H} 26 \mathrm{~A}-\mathrm{C} 26 \mathrm{~B}-\mathrm{H} 26 \mathrm{~B}$ & 109.5 \\
\hline $\mathrm{C} 18-\mathrm{C} 13-\mathrm{C} 14$ & $112.56(17)$ & $\mathrm{C} 25 \mathrm{~B}-\mathrm{C} 26 \mathrm{~B}-\mathrm{H} 26 \mathrm{C}$ & 109.5 \\
\hline $\mathrm{C} 12-\mathrm{C} 13-\mathrm{C} 14$ & $106.11(14)$ & $\mathrm{H} 26 \mathrm{~A}-\mathrm{C} 26 \mathrm{~B}-\mathrm{H} 26 \mathrm{C}$ & 109.5 \\
\hline $\mathrm{C} 18-\mathrm{C} 13-\mathrm{C} 17$ & $110.35(16)$ & $\mathrm{H} 26 \mathrm{~B}-\mathrm{C} 26 \mathrm{~B}-\mathrm{H} 26 \mathrm{C}$ & 109.5 \\
\hline $\mathrm{C} 12-\mathrm{C} 13-\mathrm{C} 17$ & $116.48(16)$ & $\mathrm{C} 25 \mathrm{~B}-\mathrm{C} 27 \mathrm{~B}-\mathrm{H} 27 \mathrm{~A}$ & 109.5 \\
\hline $\mathrm{C} 14-\mathrm{C} 13-\mathrm{C} 17$ & $99.58(14)$ & $\mathrm{C} 25 \mathrm{~B}-\mathrm{C} 27 \mathrm{~B}-\mathrm{H} 27 \mathrm{~B}$ & 109.5 \\
\hline $\mathrm{C} 8-\mathrm{C} 14-\mathrm{C} 15$ & $118.56(17)$ & $\mathrm{H} 27 \mathrm{~A}-\mathrm{C} 27 \mathrm{~B}-\mathrm{H} 27 \mathrm{~B}$ & 109.5 \\
\hline $\mathrm{C} 8-\mathrm{C} 14-\mathrm{C} 13$ & $115.10(15)$ & $\mathrm{C} 25 \mathrm{~B}-\mathrm{C} 27 \mathrm{~B}-\mathrm{H} 27 \mathrm{C}$ & 109.5 \\
\hline $\mathrm{C} 15-\mathrm{C} 14-\mathrm{C} 13$ & $104.73(15)$ & $\mathrm{H} 27 \mathrm{~A}-\mathrm{C} 27 \mathrm{~B}-\mathrm{H} 27 \mathrm{C}$ & 109.5 \\
\hline $\mathrm{C} 8-\mathrm{C} 14-\mathrm{H} 14$ & 105.8 & $\mathrm{H} 27 \mathrm{~B}-\mathrm{C} 27 \mathrm{~B}-\mathrm{H} 27 \mathrm{C}$ & 109.5 \\
\hline $\mathrm{C} 1^{\prime}-\mathrm{N} 1^{\prime}-\mathrm{N} 2^{\prime}-\mathrm{N} 3^{\prime}$ & $-0.2(2)$ & $\mathrm{C} 8-\mathrm{C} 9-\mathrm{C} 11-\mathrm{C} 12$ & $50.0(2)$ \\
\hline $\mathrm{C} 21-\mathrm{N} 1^{\prime}-\mathrm{N} 2^{\prime}-\mathrm{N} 3^{\prime}$ & $-174.88(16)$ & $\mathrm{C} 10-\mathrm{C} 9-\mathrm{C} 11-\mathrm{C} 12$ & $178.37(18)$ \\
\hline $\mathrm{N} 1^{\prime}-\mathrm{N} 2^{\prime}-\mathrm{N} 3^{\prime}-\mathrm{C} 2^{\prime}$ & $0.0(2)$ & $\mathrm{C} 9-\mathrm{C} 11-\mathrm{C} 12-\mathrm{C} 13$ & $-55.9(2)$ \\
\hline $\mathrm{N} 2^{\prime}-\mathrm{N} 1^{\prime}-\mathrm{C} 1^{\prime}-\mathrm{C} 2^{\prime}$ & $0.2(2)$ & $\mathrm{C} 11-\mathrm{C} 12-\mathrm{C} 13-\mathrm{C} 18$ & $-64.8(2)$ \\
\hline $\mathrm{C} 21-\mathrm{N} 1^{\prime}-\mathrm{C} 1^{\prime}-\mathrm{C} 2^{\prime}$ & $174.35(17)$ & $\mathrm{C} 11-\mathrm{C} 12-\mathrm{C} 13-\mathrm{C} 14$ & $57.9(2)$ \\
\hline $\mathrm{C} 10-\mathrm{C} 1-\mathrm{C} 2-\mathrm{C} 3$ & $-59.8(4)$ & $\mathrm{C} 11-\mathrm{C} 12-\mathrm{C} 13-\mathrm{C} 17$ & $167.62(16)$ \\
\hline $\mathrm{N} 2^{\prime}-\mathrm{N} 3^{\prime}-\mathrm{C} 2^{\prime}-\mathrm{C}^{\prime}$ & $0.1(2)$ & $\mathrm{C} 7-\mathrm{C} 8-\mathrm{C} 14-\mathrm{C} 15$ & $-58.9(2)$ \\
\hline $\mathrm{N} 2^{\prime}-\mathrm{N} 3^{\prime}-\mathrm{C} 2^{\prime}-\mathrm{C}^{\prime}$ & $179.24(18)$ & $\mathrm{C} 9-\mathrm{C} 8-\mathrm{C} 14-\mathrm{C} 15$ & $-178.86(17)$ \\
\hline $\mathrm{N1}^{\prime}-\mathrm{C} 1^{\prime}-\mathrm{C} 2^{\prime}-\mathrm{N}^{\prime}$ & $-0.2(2)$ & $\mathrm{C} 7-\mathrm{C} 8-\mathrm{C} 14-\mathrm{C} 13$ & $176.05(17)$ \\
\hline
\end{tabular}




\begin{tabular}{|c|c|c|c|}
\hline $\mathrm{N} 1^{\prime}-\mathrm{C} 1^{\prime}-\mathrm{C} 2^{\prime}-\mathrm{C} 3^{\prime}$ & $-179.2(2)$ & $\mathrm{C} 9-\mathrm{C} 8-\mathrm{C} 14-\mathrm{C} 13$ & $56.1(2)$ \\
\hline $\mathrm{C} 1-\mathrm{C} 2-\mathrm{C} 3-\mathrm{O} 3$ & $177.9(3)$ & $\mathrm{C} 18-\mathrm{C} 13-\mathrm{C} 14-\mathrm{C} 8$ & $61.5(2)$ \\
\hline $\mathrm{C} 1-\mathrm{C} 2-\mathrm{C} 3-\mathrm{C} 4$ & $54.9(4)$ & $\mathrm{C} 12-\mathrm{C} 13-\mathrm{C} 14-\mathrm{C} 8$ & $-60.4(2)$ \\
\hline $\mathrm{N} 3^{\prime}-\mathrm{C} 2^{\prime}-\mathrm{C} 3^{\prime}-\mathrm{O} 3^{\prime}$ & $173.26(18)$ & $\mathrm{C} 17-\mathrm{C} 13-\mathrm{C} 14-\mathrm{C} 8$ & $178.33(16)$ \\
\hline $\mathrm{C} 1^{\prime}-\mathrm{C} 2^{\prime}-\mathrm{C} 3^{\prime}-\mathrm{O} 3^{\prime}$ & $-7.8(3)$ & $\mathrm{C} 18-\mathrm{C} 13-\mathrm{C} 14-\mathrm{C} 15$ & $-70.5(2)$ \\
\hline $\mathrm{N} 3^{\prime}-\mathrm{C} 2^{\prime}-\mathrm{C} 3^{\prime}-\mathrm{C} 4^{\prime}$ & $-67.0(3)$ & $\mathrm{C} 12-\mathrm{C} 13-\mathrm{C} 14-\mathrm{C} 15$ & $167.64(17)$ \\
\hline $\mathrm{C} 1^{\prime}-\mathrm{C} 2^{\prime}-\mathrm{C} 3^{\prime}-\mathrm{C} 4^{\prime}$ & $111.9(3)$ & $\mathrm{C} 17-\mathrm{C} 13-\mathrm{C} 14-\mathrm{C} 15$ & $46.35(19)$ \\
\hline $\mathrm{N} 3^{\prime}-\mathrm{C} 2^{\prime}-\mathrm{C} 3^{\prime}-\mathrm{C} 5^{\prime}$ & $54.7(3)$ & $\mathrm{C} 8-\mathrm{C} 14-\mathrm{C} 15-\mathrm{C} 16$ & $-162.71(18)$ \\
\hline $\mathrm{C} 1^{\prime}-\mathrm{C} 2^{\prime}-\mathrm{C} 3^{\prime}-\mathrm{C} 5^{\prime}$ & $-126.4(3)$ & $\mathrm{C} 13-\mathrm{C} 14-\mathrm{C} 15-\mathrm{C} 16$ & $-32.7(2)$ \\
\hline $\mathrm{O} 3-\mathrm{C} 3-\mathrm{C} 4-\mathrm{C} 5$ & $-175.9(2)$ & $\mathrm{C} 14-\mathrm{C} 15-\mathrm{C} 16-\mathrm{C} 17$ & $5.9(2)$ \\
\hline $\mathrm{C} 2-\mathrm{C} 3-\mathrm{C} 4-\mathrm{C} 5$ & $-51.3(3)$ & $\mathrm{C} 15-\mathrm{C} 16-\mathrm{C} 17-\mathrm{C} 20$ & $150.76(18)$ \\
\hline $\mathrm{C} 3-\mathrm{C} 4-\mathrm{C} 5-\mathrm{C} 6$ & $-128.2(3)$ & $\mathrm{C} 15-\mathrm{C} 16-\mathrm{C} 17-\mathrm{C} 13$ & $22.5(2)$ \\
\hline $\mathrm{C} 3-\mathrm{C} 4-\mathrm{C} 5-\mathrm{C} 10$ & $51.2(3)$ & $\mathrm{C} 18-\mathrm{C} 13-\mathrm{C} 17-\mathrm{C} 20$ & $-48.0(2)$ \\
\hline $\mathrm{C} 4-\mathrm{C} 5-\mathrm{C} 6-\mathrm{C} 7$ & $-177.4(2)$ & $\mathrm{C} 12-\mathrm{C} 13-\mathrm{C} 17-\mathrm{C} 20$ & $80.0(2)$ \\
\hline $\mathrm{C} 10-\mathrm{C} 5-\mathrm{C} 6-\mathrm{C} 7$ & $3.2(4)$ & $\mathrm{C} 14-\mathrm{C} 13-\mathrm{C} 17-\mathrm{C} 20$ & $-166.57(17)$ \\
\hline $\mathrm{C} 5-\mathrm{C} 6-\mathrm{C} 7-\mathrm{C} 8$ & $14.6(3)$ & $\mathrm{C} 18-\mathrm{C} 13-\mathrm{C} 17-\mathrm{C} 16$ & $77.23(19)$ \\
\hline $\mathrm{C} 6-\mathrm{C} 7-\mathrm{C} 8-\mathrm{C} 14$ & $-165.92(18)$ & $\mathrm{C} 12-\mathrm{C} 13-\mathrm{C} 17-\mathrm{C} 16$ & $-154.78(16)$ \\
\hline $\mathrm{C} 6-\mathrm{C} 7-\mathrm{C} 8-\mathrm{C} 9$ & $-45.3(2)$ & $\mathrm{C} 14-\mathrm{C} 13-\mathrm{C} 17-\mathrm{C} 16$ & $-41.30(18)$ \\
\hline $\mathrm{C} 14-\mathrm{C} 8-\mathrm{C} 9-\mathrm{C} 11$ & $-48.2(2)$ & $\mathrm{C} 16-\mathrm{C} 17-\mathrm{C} 20-\mathrm{C} 21$ & $53.8(2)$ \\
\hline $\mathrm{C} 7-\mathrm{C} 8-\mathrm{C} 9-\mathrm{C} 11$ & $-169.37(17)$ & $\mathrm{C} 13-\mathrm{C} 17-\mathrm{C} 20-\mathrm{C} 21$ & $174.09(17)$ \\
\hline $\mathrm{C} 14-\mathrm{C} 8-\mathrm{C} 9-\mathrm{C} 10$ & $-176.74(16)$ & $\mathrm{C} 16-\mathrm{C} 17-\mathrm{C} 20-\mathrm{C} 22$ & $178.58(17)$ \\
\hline $\mathrm{C} 7-\mathrm{C} 8-\mathrm{C} 9-\mathrm{C} 10$ & $62.1(2)$ & $\mathrm{C} 13-\mathrm{C} 17-\mathrm{C} 20-\mathrm{C} 22$ & $-61.1(2)$ \\
\hline $\mathrm{C} 6-\mathrm{C} 5-\mathrm{C} 10-\mathrm{C} 19$ & $-111.7(3)$ & $\mathrm{N} 2{ }^{\prime}-\mathrm{N} 11^{\prime}-\mathrm{C} 21-\mathrm{C} 20$ & $59.8(2)$ \\
\hline $\mathrm{C} 4-\mathrm{C} 5-\mathrm{C} 10-\mathrm{C} 19$ & $68.8(3)$ & $\mathrm{C} 1^{\prime}-\mathrm{N} 1{ }^{\prime}-\mathrm{C} 21-\mathrm{C} 20$ & $-113.9(2)$ \\
\hline $\mathrm{C} 6-\mathrm{C} 5-\mathrm{C} 10-\mathrm{C} 9$ & $11.8(3)$ & $\mathrm{C} 22-\mathrm{C} 20-\mathrm{C} 21-\mathrm{N} 1^{\prime}$ & $49.7(2)$ \\
\hline $\mathrm{C} 4-\mathrm{C} 5-\mathrm{C} 10-\mathrm{C} 9$ & $-167.7(2)$ & $\mathrm{C} 17-\mathrm{C} 20-\mathrm{C} 21-\mathrm{N} 1^{\prime}$ & $176.60(17)$ \\
\hline $\mathrm{C} 6-\mathrm{C} 5-\mathrm{C} 10-\mathrm{C} 1$ & $128.9(2)$ & $\mathrm{C} 21-\mathrm{C} 20-\mathrm{C} 22-\mathrm{C} 23$ & $63.9(2)$ \\
\hline $\mathrm{C} 4-\mathrm{C} 5-\mathrm{C} 10-\mathrm{C} 1$ & $-50.6(3)$ & $\mathrm{C} 17-\mathrm{C} 20-\mathrm{C} 22-\mathrm{C} 23$ & $-60.0(2)$ \\
\hline $\mathrm{C} 11-\mathrm{C} 9-\mathrm{C} 10-\mathrm{C} 5$ & $-172.73(18)$ & $\mathrm{C} 20-\mathrm{C} 22-\mathrm{C} 23-\mathrm{C} 24 \mathrm{~B}$ & $157.7(7)$ \\
\hline $\mathrm{C} 8-\mathrm{C} 9-\mathrm{C} 10-\mathrm{C} 5$ & $-44.4(2)$ & $\mathrm{C} 20-\mathrm{C} 22-\mathrm{C} 23-\mathrm{C} 24$ & $171.5(2)$ \\
\hline $\mathrm{C} 11-\mathrm{C} 9-\mathrm{C} 10-\mathrm{C} 19$ & $-50.3(3)$ & $\mathrm{C} 22-\mathrm{C} 23-\mathrm{C} 24-\mathrm{C} 25$ & $176.0(3)$ \\
\hline $\mathrm{C} 8-\mathrm{C} 9-\mathrm{C} 10-\mathrm{C} 19$ & $78.0(2)$ & $\mathrm{C} 23-\mathrm{C} 24-\mathrm{C} 25-\mathrm{C} 27$ & $-73.4(4)$ \\
\hline $\mathrm{C} 11-\mathrm{C} 9-\mathrm{C} 10-\mathrm{C} 1$ & $71.0(2)$ & $\mathrm{C} 23-\mathrm{C} 24-\mathrm{C} 25-\mathrm{C} 26$ & $56.4(5)$ \\
\hline $\mathrm{C} 8-\mathrm{C} 9-\mathrm{C} 10-\mathrm{C} 1$ & $-160.65(18)$ & $\mathrm{C} 22-\mathrm{C} 23-\mathrm{C} 24 \mathrm{~B}-\mathrm{C} 25 \mathrm{~B}$ & $171.1(8)$ \\
\hline $\mathrm{C} 2-\mathrm{C} 1-\mathrm{C} 10-\mathrm{C} 5$ & $55.4(3)$ & $\mathrm{C} 23-\mathrm{C} 24 \mathrm{~B}-\mathrm{C} 25 \mathrm{~B}-\mathrm{C} 26 \mathrm{~B}$ & $158.2(11)$ \\
\hline $\mathrm{C} 2-\mathrm{C} 1-\mathrm{C} 10-\mathrm{C} 19$ & $-63.7(3)$ & $\mathrm{C} 23-\mathrm{C} 24 \mathrm{~B}-\mathrm{C} 25 \mathrm{~B}-\mathrm{C} 27 \mathrm{~B}$ & $-65.1(15)$ \\
\hline $\mathrm{C} 2-\mathrm{C} 1-\mathrm{C} 10-\mathrm{C} 9$ & $174.2(2)$ & & \\
\hline
\end{tabular}

Hydrogen-bond geometry $\left(\AA,{ }^{\circ}\right)$

\begin{tabular}{lllll}
\hline$D-\mathrm{H}^{\prime} \cdots A$ & $D-\mathrm{H}$ & $\mathrm{H} \cdots A$ & $D \cdots A$ & $D-\mathrm{H}^{\cdots} \cdots A$ \\
\hline $\mathrm{O} 3-\mathrm{H} 3 \cdots 3^{\prime i \mathrm{i}}$ & 0.84 & 2.00 & $2.811(3)$ & 162 \\
$\mathrm{O}^{\prime}-\mathrm{H}^{\prime} \cdots \mathrm{N}^{\prime}{ }^{\prime i \mathrm{i}}$ & 0.84 & 1.97 & $2.810(2)$ & 175 \\
\hline
\end{tabular}

Symmetry codes: (i) $x+1 / 2, y+1 / 2, z+1$; (ii) $-x-1 / 2, y-1 / 2,-z$. 